\title{
RESEARCH
}

Open Access

\section{Essential role of ATP6AP2 enrichment in caveolae/lipid raft microdomains for the induction of neuronal differentiation of stem cells}

Nehman Makdissy ${ }^{1 *}$ (D) Katia Haddad ${ }^{1 \dagger}$, Jeanne D'arc AlBacha ${ }^{2 \dagger}$, Diana Chaker ${ }^{2}$, Bassel Ismail ${ }^{3}$, Albert Azar ${ }^{4}$, Ghada Oreibi ${ }^{4}$, David Ayoub ${ }^{5}$, Ibrahim Achkar ${ }^{6}$, Didier Quilliot ${ }^{7}$ and Ziad Fajloun ${ }^{1,2}$

\begin{abstract}
Background: The subcellular distribution of prorenin receptor and adaptor protein ATP6AP2 may affect neurogenesis. In this study, we hypothesized that ATP6AP2 expression and subcellular relocalization from caveolae/lipid raft microdomains (CLR-Ms) to intracellular sites may correlate with neuronal differentiation (Neu-Dif) of adipose-derived mesenchymal stem cells (ADSCS).

Methods: Human ADSCs isolated from 24 healthy donors and 24 patients with neurological disorders (ND) were cultured and induced for Neu-Dif. The mechanism of action of ATP6AP2 and the impact of its localization within the plasma membrane (particularly CLR-Ms) and intracellular sites on several pathways (mitogen-activated protein kinase, Wnt(s) signaling and others) and intracellular calcium and exosome release were evaluated. The impact of CLR-Ms on ATP6AP2 or vice versa was determined by pharmacological disruption of CLR-Ms or SIATP6AP2 assays.

Results: In patients with ND, loss of ATP6AP2 from CLR-Ms correlated with an inhibition of Neu-Dif and signaling. However, its relocalization in CLR-Ms was positively correlated to induction of Neu-Dif in healthy subjects. An apparent switch from canonical to noncanonical Wnt signaling as well as from caveolin to flotillin occurs concurrently with the increases of ATP6AP2 expression during neurogenesis. Stimulation by renin activates ERK/JNK/CREB/C-Jun but failed to induce $\beta$-catenin. Wnt5a enhanced the renin-induced JNK responsiveness. Ga proteins crosslink ATP6AP2 to caveolin where a switch from Gai to Gaq is necessary for Neu-Dif. In ATP6AP2-enriched CLR-Ms, the release of exosomes was induced dependently from the intracellular $\mathrm{Ca}^{2+}$ and Gaq. Pharmacological disruption of CLR-M formation/stability impairs both ATP6AP2 localization and Neu-Dif in addition to reducing exosome release, indicating an essential role of ATP6AP2 enrichment in CLR-Ms for the induction of Neu-Dif. The mechanism is dependent on CLR-M dynamics, particularly the membrane fluidity. Knockdown of ATP6AP2 inhibited Neu-Dif but increased astrocytic-Dif, depleted ATP6AP2/flotillin/Gaq but accumulated caveolin/Gai in CLR-Ms, and blocked the activation of JNK/ERK/c-Jun/CREB/exosome release. siATP6AP2 cells treated with sphingomyelinase/methyl- $\beta$ cyclodextrin reversed the levels of caveolin/flotillin in CLR-Ms but did not induce Neu-Dif, indicating the crucial relocalization of ATP6AP2 in CLR-Ms for neurogenesis. Treatment of ND-derived cells with nSMase showed reversibility in ATP6AP2 abundance in CLR-Ms and enhanced Neu-Dif.

(Continued on next page)
\end{abstract}

\footnotetext{
* Correspondence: almakdissy@hotmail.com

${ }^{\dagger}$ Equal contributors

'Department of Biology, Lebanese University, Faculty of Sciences III, Kobbe,

Lebanon

Full list of author information is available at the end of the article
}

(c) The Author(s). 2018 Open Access This article is distributed under the terms of the Creative Commons Attribution 4.0 International License (http://creativecommons.org/licenses/by/4.0/), which permits unrestricted use, distribution, and reproduction in any medium, provided you give appropriate credit to the original author(s) and the source, provide a link to the Creative Commons license, and indicate if changes were made. The Creative Commons Public Domain Dedication waiver (http://creativecommons.org/publicdomain/zero/1.0/) applies to the data made available in this article, unless otherwise stated. 
(Continued from previous page)

Conclusions: This study gives evidence of the determinant role of CLR-M ATP6AP2 localization for neuronal and oligodendrocyte differentiation involving mechanisms of switches from Gai/caveolin/canonical to Gaq/ flotillin/PCP, the ERK/JNK pathway and $\mathrm{Ca}^{2+}$-dependent release of exosomes and as a potential target of drug therapy for neurodegenerative disorders.

Keywords: ATP6AP2, Caveolae, Caveolin, Exosomes, Flotillin, Lipid rafts, Wnt signaling, Neural differentiation, Renin, Stem cells

\section{Background}

MSCs are multipotent stromal cells with the potential to differentiate into neural cells, involving several signaling pathways. An important role for expression of the renin-angiotensin system (RAS) has been reported in the regulation of human MSC differentiation [1]. ATP6AP2 was described as a renin and prorenin receptor $((\mathrm{P}) \mathrm{RR})$ exerting a RAS-related function but also as an adaptor protein between V-ATPase and Wnt receptor complex [2]. Binding of prorenin or renin to the extracellular domain of ATP6AP2 activates the RAS, leading to the production of angiotensin II and other pathways, such as the MAPK pathway, in particular ERK [3]. The beginning of the discovery of ATP6AP2 as an essential component for RAS was followed by that of its expression in the brain $[4,5]$. In XPDS patients, a decrease of ATP6AP2 was observed in the brain [6]. In the mouse and fly, conditional depletion of ATP6AP2 induces cognitive impairment and neurodegeneration [7], and regulation of adult hippocampal neurogenesis via Wnt/PCP/ $\beta$-catenin pathways. In Xenopus, ATP6AP2 is essential for mediating Wnt signaling during early central nervous system development, including neural patterning [8]. Thereby, emerging studies have drawn attention to the importance of ATP6AP2 in cognitive processes and brain development while suggesting a vital role of the RAS in learning and memory functions. However, little is known about the importance of the colocalization of ATP6AP2 with key signaling factors, and even rarer are studies about its relocalization and its relationship with plasma membrane microdomains particularly in MSCs.

Previous studies underline the combination role of ATP6AP2 and other factors, among them, for example, vascular endothelial growth factor (VEGF), a growth factor secreted by MSCs [9], known to possess neurogenic effects, promote NSC activation and regulate their conversion into progenitor cells and neurons [10]. Importantly, colocalization of ATP6AP2 and VEGF has been reported in human retinal microvascular endothelial cells [11]. Moreover, ATP6AP2 interacting with microRNA-152 (a potential regulator of ATP6AP2) regulates downstream VEGF expression [12]. Recent evidence has also shown that neuronal differentiation (Neu-Dif) of neural progenitor cells can be regulated by caveolin (CAV) proteins, components of the microdomain lipid rafts (MLRs), via modulation of a variety of neuronal intracellular signaling pathways, among them VEGF, ERK, Akt and Stat3 [13, 14]. All three CAV isoforms are expressed in neurons and immature neuronal-like cells lose CAV expression upon differentiation [15-18]. In fact, MLRs enriched in sphingolipids, cholesterol, scaffolding proteins and a multitude of receptors including neurotransmitter receptors serve as a platform for neuronal signal transduction and cytoskeletal organization by interaction with CAV [19]. Among the proteins in MLRs, the integral membrane flotillin (FLOT) may represent a functional analogue of CAV in CAV-negative or downregulated cells; in fact, FLOT is highly expressed in cells that lack CAV (e.g., neurons) [20]. FLOT-1/FLOT-2 define raft-related microdomain signaling centers in neurons and astrocytes [21]. Any perturbation in MLRs can affect the cell autophagy or the release of exosomes affecting FLOTs and CAVs, and may have significant impacts on Wnt signaling as well as secreted inflammatory cytokines [22, 23]. In addition, several lines of evidence suggest that V-ATPase is a major component of the MLR fraction of synaptic plasma membrane and synaptic vesicle membrane [24].

Taking into consideration the potential role of ATP6AP2 in neuronal development, several points remain to be clarified concerning its role in MSCs during neurogenesis: ATP6AP2 has not yet been identified in MSCs, and the subcellular distribution of (P)RR in MSCs and the relationship with MLRs remain to be elucidated. Therefore, we were interested in this study to isolate human adiposederived mesenchymal stem cells (hADSCs) and to evaluate the relationship between ATP6AP2 and these microdomains, studying the mechanisms involving Wnt/Go proteins/intracellular calcium/exosome release signaling pathways. The role of ATP6AP2 was validated with the use of gene knockdown ADSCs. A neurological diseases group of patients was compared to a healthy group of subjects.

\section{Methods}

\section{Subjects}

Healthy subjects $(n=24,57.8 \pm 3.1$ years old $)$ were enrolled in the study if they had no notable pathologic history in particular neurological diseases. Subjects with confirmed diagnosis of ND $(n=24,58.9 \pm 3.4$ years old $)$ 
(Alzheimer's disease (AD), $n=6$; Parkinson's disease (PD), $n=9$; amyotrophic lateral sclerosis (ALS), $n=4$; multiple sclerosis (MS), $n=5$ ) were considered for this study as ND patients. The selection of subjects' age was group matched (healthy vs ND) (Fig. 1). The control group consisted of healthy volunteers matched to the ND groups for age and sex. Subjects were excluded from the two groups if they had histories of neurologic conditions including moderate or severe head injury, stroke, cerebral or bone damage or malignancies, brain abnormalities, learning disability, major medical or psychiatric illness in the previous 6 months, any metabolic/cardiovascular disease or evidence of cardiac/renal damage or malignancies, alcohol, loss of weight during the last 2 years, chemotherapy or immunosuppressive therapy.

\section{Isolation and culture of peripheral blood mononuclear cells}

Fresh peripheral blood $(20 \mathrm{ml})$ was collected from healthy and ND subjects. Peripheral blood mononuclear cells (PBMCs) were separated and cultured according to AlBacha et al. [25]. No significant variations on the number of isolated cells were obtained between healthy and ND subjects (data not shown).

\section{Isolation and culture of hADSCs}

Samples of human adipose tissue $(200 \mathrm{ml}$ or $\sim 100-$ $200 \mathrm{mg}$ ) were obtained by lipoaspiration or biopsy from abdominal subcutaneous fat, and then processed for the isolation of SVF and culture of ADSCs as described previously [26]. The differentiation was induced at the 3rd passage after confirming the absence of any chromosomal abnormality as determined by karyotyping. No significant variations in the number of isolated cells were obtained between healthy and ND subjects (Fig. 1).

\section{Neuronal induction of hMSCs}

This procedure consisted of two steps: induction of hMSCs to differentiate into neurosphere-like structures (NSPs), and final differentiation into neuron-like cells (NLCs). MSCs $\left(10^{6}\right.$ cells) were cultured in a neurobasal (NB) medium (Hyclone advanced basal medium stem) supplemented with $10 \%$ serum (Hyclone advanced stem cell growth supplement), $2 \%$ B27, $1 \%$ PSA at $37{ }^{\circ} \mathrm{C}$ and $5 \% \mathrm{CO}_{2}$ (days 0-14). NB medium was supplemented with $20 \mathrm{ng} / \mathrm{ml} \mathrm{b-FGF}$ and $20 \mathrm{ng} / \mathrm{ml}$ EGF for 16 days (days 14-30). Formed NSPs were collected at day 30 and then cultured to initiate final differentiation. NSPs $\left(10^{5}\right.$ cells) were cultured in (NB) medium supplemented with $10 \%$ serum, $2 \%$ B27, 1\% PSA, $20 \mathrm{ng} / \mathrm{ml} \beta-\mathrm{NGF}$ at $37{ }^{\circ} \mathrm{C}$ and $5 \% \mathrm{CO}_{2}$ (days 30-37). Media were renewed every 3-4 days.
Dosage of soluble (pro)renin receptor by ELISA

Soluble (pro)renin receptor $(\mathrm{s}(\mathrm{P}) \mathrm{RR})$ was quantified using an ELISA kit (Immuno-Biological Laboratories) according to the instructions of the manufacturer. $s(\mathrm{P})$ RR was detected by an HRP-conjugated antibody and TMB as chromogen.

\section{Membrane fluidity}

The plasma membrane fluidity was assessed by steadystate fluorescence polarization using the lipophilic fluorescent probe DPH. The measured fluorescence anisotropy, inversely related to fluidity, was determined as previously described by Makdissy et al. [27].

\section{Cell fractionation and isolation of caveolae/lipid rafts}

Caveolae/lipid raft MLRs were prepared according to the method of Makdissy et al. [27] adapted for MSCs, NSPs and NLCs. In total, 12-13 fractions were obtained, subdivided into: nuclear fraction, postnuclear fraction (which was depleted from nuclear and plasma membranes), microsomal fraction and plasma membranes (total or fractioned into the caveolae/lipid raft plasma membrane microdomain (CLR-M) fraction and the noncaveolae/nonlipid raft plasma membrane microdomain (N-CLR-M) fraction). To ensure the absence of nuclear and microsomal membrane contamination in the plasma membrane fraction, the obtained fractions were analyzed for the presence of nuclear protein nucleoporin p62 and largely Golgi-localized protein TGN38 (H-122 (sc-25523) and C-15 (sc-27680)) (Santa Cruz) (Additional file 1: Table SI1).

\section{Cell fractionation and isolation of intracellular organelles}

After isolation of cytoplasmic and nuclear fractions from cultured cells, the intracellular organelle fraction was isolated by OptiPrep density gradient and sucrose. The isolation of endoplasmic reticulum (ER) Golgi and lysosome fractions (EL0100, GL0010, and LYSISO1, respectively; Sigma) was performed according to the manufacturer's instructions. Organelle markers were used to identify the intracellular components: ER (Calnexin, TRAP $\alpha$ ), Golgi (TGN38, GM130), endosome (EEA1), lysosome (LAMP1) and nuclear (Nucleoporin, Lamin A).

\section{Exosome purification}

Exosomes were isolated from the culture media of $10^{7}$ cells according to the method described previously by Théry et al. [28]. The purity of the exosomes was evaluated by flow cytometry determining the percentages of expression of specific tetraspanin exosome biomarkers (CD9, CD63 and CD81) and FLOT, and the absence of the ER marker (calnexin). Released exosomes were quantitated by measuring the activity of their specific enzyme: 


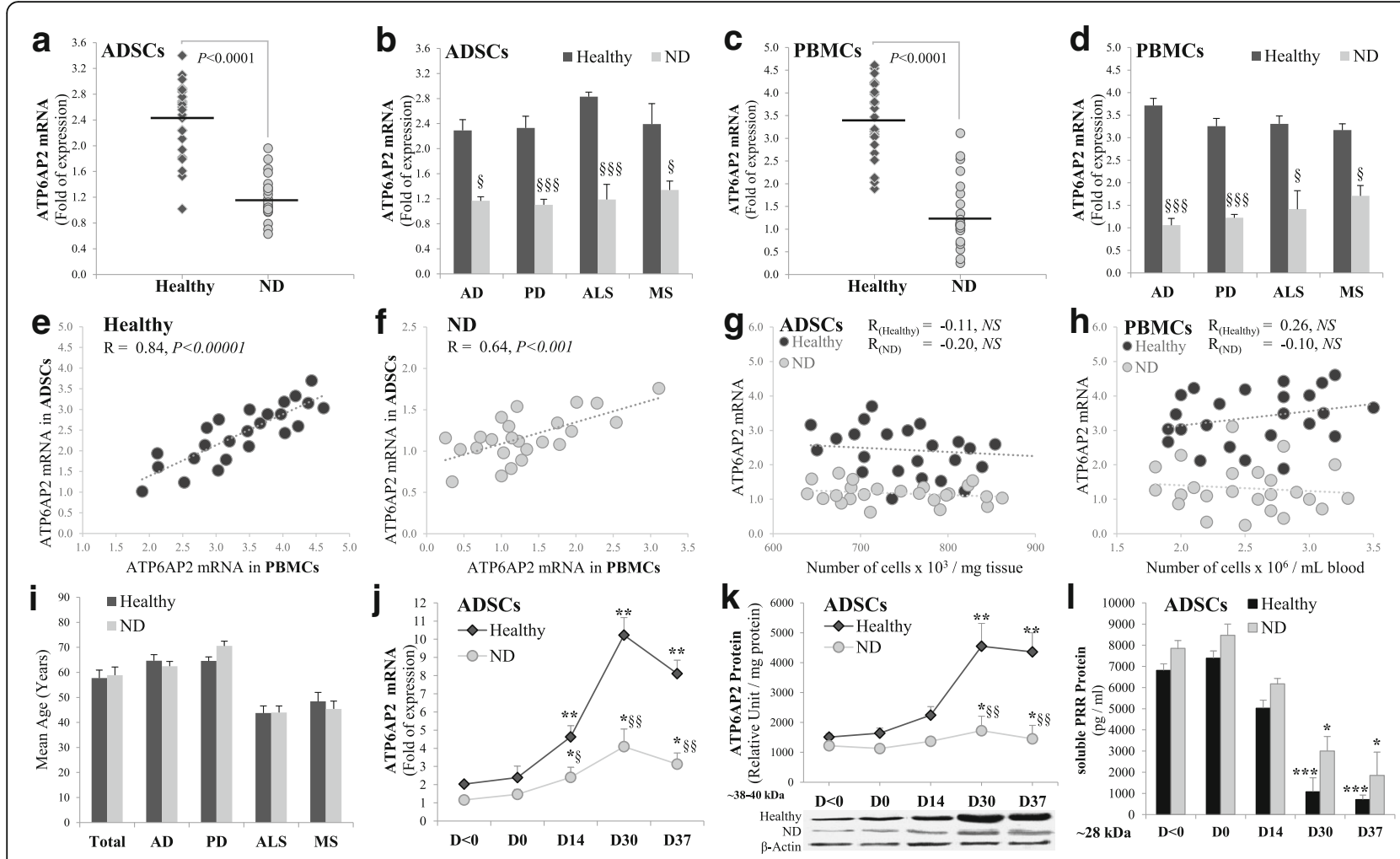

Fig. 1 Expression of ATP6AP2 in PBMCs and ADSCs. PBMCs and ADSCs isolated from healthy or ND subjects. mRNA expression determined by RT-qPCR and normalized to GAPDH. Quantitative levels of ATP6AP2 mRNA in undifferentiated ADSCs (a, $\mathbf{b})$ or PBMCs (c, $\mathbf{d})$. Correlations of expression of ATP6AP2 between ADSCs and PBMCs in healthy (e) or ND (f) subjects. Correlations with isolated number of cells in ADSCs (g) or PBMCs (h). Distribution of population as a function of donor's age (i). ADSCs differentiated into NLCs for 37 days as indicated in Methods and levels of mRNA (j), total (k) and soluble (I) proteins shown. Undifferentiated cells analyzed at day $<0(\mathbf{a}-\mathbf{h})$. Differentiated cells analyzed at $\mathrm{D}<0 / \mathrm{D} 0 / \mathrm{D} 14 / \mathrm{D} 30 / \mathrm{D} 37(\mathbf{j}-\mathbf{I}) .{ }^{* \$} P<$ $0.05,{ }^{* * \S \S} P<0.001,{ }^{* * * \$ \S \S} P<0.0005$ : ${ }^{*} D 0 / 14 / 30 / 37$ vs $D<0,{ }^{\S} \mathrm{ND}$ vs healthy. AD Alzheimer disease, ADSC adipose-derived mesenchymal stem cell, ALS amyotrophic lateral sclerosis, D day, MS multiple sclerosis, ND neurodegenerative disease, NS not significant, PBMC peripheral blood mononuclear cell, PD, Parkinson disease

acetylcholinesterase (AChE). The exosome fraction was suspended in PBS (1:4, v:v) and incubated with DTNB $(0.1 \mathrm{mM})$ and acetylthiocholine $(1.25 \mathrm{mM})$ in a final volume of $1 \mathrm{ml}$ at $37{ }^{\circ} \mathrm{C}$, and the change in absorbance at $412 \mathrm{~nm}$ was determined continuously at 10/20/30/60/180 min. The data represent the enzymatic activity at $30 \mathrm{~min}$ of incubation (saturation reached its maximum at $20 \mathrm{~min}$ ).

\section{Magnetic separation of $\mathrm{TUJ1}^{(+)} / \mathrm{O} 4^{(+)} / \mathrm{GFAP}^{(+)}$cells}

hADSCs derived from healthy and ND subjects were cultured and differentiated as described previously. Purifications of $\mathrm{TUJ}^{(+)} / \mathrm{O}^{(+)} / \mathrm{GFAP}^{(+)}$cells were performed on the mixed population at day $<0$ (before the induction of the differentiation) and at day 37 (end of the NeuDif). Then, $20 \times 10^{7}$ total cells were collected and subjected to purification using MACS technology (Miltenyi) according to the manufacturer's instructions. Cells were stained for $30 \mathrm{~min}$ in the dark with the corresponding fluorochrome-conjugated primary antibody (monoclonal anti-TUBB3-FITC antibody (for TUJ1 staining; Sigma-Aldrich), monoclonal anti-O4-APC antibody (for $\mathrm{O} 4$ staining; Miltenyi) and monoclonal anti-GFAP-PE antibody (for GFAP staining; Miltenyi)); a first measure on a sample of the mixed population was done by flow cytometry for determination of the percentage of cells which may express these markers. Therefore, we proceeded with targeted-cell purification for $15 \mathrm{~min}$ at $4{ }^{\circ} \mathrm{C}$ : to obtain a single-cell suspension before magnetic labeling, cells were passed through $70-\mu \mathrm{m}$ nylon mesh (Miltenyi) to remove cell clumps which may clog the column. Subsequently, the obtained single-cell suspension was magnetically labeled firstly with antiFITC Microbeads (Miltenyi) for the purification of TUJ1. The cell suspension was loaded on a MACS column which was placed in the magnetic field of a MACS separator. The magnetically labeled $\mathrm{TUJ}^{(+)}$cells were retained in the column while the unlabeled cells ran through (negative fraction designated $\mathrm{N} 1$ : this cell fraction was thus depleted from $\left.\mathrm{TUJ}^{(+)}\right)$. After removing the column from the magnetic field, the magnetically retained $\mathrm{TUJ}^{(+)}$cells were eluted as the positively selected $\mathrm{TUJ}^{(+)}$cell fraction (positive fraction 
designated P1). Next, the negative fraction N1 was then labeled with anti-APC Microbeads (Miltenyi) for the purification of $\mathrm{O} 4$; the magnetically labeled $\mathrm{O}^{(+)}$cells were purified as described previously to obtain a P2 fraction, while the unlabeled cells constituted the negative fraction N2 which was depleted of $\mathrm{TUJ}^{(+)}$and $\mathrm{O}^{(+)}$. Finally, N2 was labeled with anti-PE Microbeads (Miltenyi) for purification of GFAP; the same procedure and elution as described previously was used to obtain P3 enriched with GFAP $^{(+)}$cells. To increase the purity, the positively selected cell fractions were separated over a second column. After fluorescent labeling, samples of the obtained positive and negative fractions were reanalyzed by flow cytometry to assess purity and analyze the immunophenotyping of the cells.

\section{Flow cytometry analysis}

Cells were stained with human anti-CD34(Vioblue), CD45(FITC), CD73(PE), CD90(APC) and CD105(Vioblue) antibodies (Miltenyi-Biotech), TUJ1 (Covance or Sigma-Aldrich), NeuN and GFAP (Abcam or Miltenyi) and O4 (Sigma). Purified exosomes were fixed and incubated with human anti-CD9(PE), anti-CD63(APC) and anti-CD81(PerCP) antibodies (Miltenyi), FLOT-1/FLOT2 and $\beta$-catenin (Santa-Cruz) and calnexin (Abcam). Appropriate APC/FITC/PE-conjugated secondary antibodies were used in cases of staining with primary unconjugated antibodies. The flow cytometry protocol and analysis was performed following supplier instructions on an MACSQuant analyzer (Miltenyi). Isotype controls and automated compensation were settled to minimize false positive fluorescence and spectral overlap of fluorochromes respectively. Cell viability and apoptosis were assessed by the 7AAD/AnnexinV/PI assay.

\section{Immunostaining and fluorescence microscopy analysis}

At day 37 of Neu-Dif, cells were seeded on round glass coverslips coated with poly-L-lysine $(0.01 \%)$, fixed in $4 \%$ paraformaldehyde $(20 \mathrm{~min}$ ) and then washed twice with PBS (5 min). Three sequential immunostainings were performed with three different antibodies: TUJ1, GFAP and O4. Immunostaining was performed after blocking in 3\% normal goat serum and adding primary mouse monoclonal TUJ1 (1/1000), rabbit polyclonal IgG anti-GFAP (1/800) and mouse monoclonal anti-O4 (1/1000). The secondary antibodies were ATTO $\alpha$-mouse IgG Alexa 488 (1/500), AHO $647 \alpha$-rabbit (1/500) and mouse IgM 488 (1/1000). Slides were treated with VECTASHIELD mounting Medium with DAPI and viewed on a SP5 inverted confocal microscope. Images were captured and analyzed on Leica software.

\section{RT-qPCR}

RNA was isolated with the RNAspin Mini kit (GE Healthcare) according to the manufacturer's instructions and
DNase digestion was performed with the RNase-Free DNase Set (Qiagen). The yield and purity of RNA were assessed spectrophotometrically and the integrity by agarose gel electrophoresis. First-strand cDNA was synthesized from $400 \mathrm{ng}$ RNA using Superscript III reverse transcriptase (Invitrogen) according to the manufacturer's protocol. cDNA (25 ng) relative to total RNA was amplified in $20 \mu \mathrm{l}$ using VeriQuest Fast SYBR Green qPCR Master Mix (Affymetrix). Thermal cycling was performed on a LightCycler 2.0 (Roche): 1 cycle at $50{ }^{\circ} \mathrm{C} / 2 \mathrm{~min}$, 1 cycle at $95^{\circ} \mathrm{C} / 5 \mathrm{~min}, 45$ cycles at $95^{\circ} \mathrm{C} / 3 \mathrm{~s}$ and $60{ }^{\circ} \mathrm{C} / 30$ s. The primers are presented in Additional file 2: Table SI2. Relative changes in expression were calculated after normalization to GAPDH.

\section{ATP6AP2 siRNA transfection}

MSCs at $70 \%$ of confluence were cultured $\left(10^{5}\right.$ cells $)$ and transfected with Lipofectamine RNAiMAX (Thermofisher Scientific) according to the manufacturer's instructions. MSCs were transfected with siRNA-Lipofectamine RNAiMAX complexes (respectively, $6000 \mathrm{pmol}$ and $40 \mu \mathrm{l}$ in $2 \mathrm{ml}$ media) at day $<0$ and the cells were induced to differentiate with the neuronal induction medium. To maintain the silencing effect, an additional dose of siRNAs was administered $7 / 14 / 28$ days after the initial transfection. Silencing was validated by RT-qPCR. Cytotoxic effects were observed above $11,000 \mathrm{pmol}$ of siRNA and if added with medium supplemented with antibiotics and high concentrations of serum (>4\%). Internal control of lipofectamine alone was used.

\section{Western blot analysis}

The proteins were revealed with antibodies directed against ATP6AP2(HPA003156), ERK(sc-94), p-ERK(sc-7383), JN K(FL,sc-571), p-JNK(G-7,sc-6254), CREB-1(sc-58), p-CREB1(sc-7978), c-Jun(sc-74,543), p-c-Jun(sc-822), $\beta$-catenin(c-72 04, sc-7963), caveolin(sc-894), flotillin(sc-30,750), Gas/

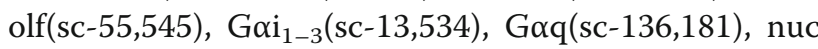
leoporin p62(sc-25,523) and TGN38(sc-27,680). SDSPAGE analysis was performed as described previously by Makdissy et al. [27].

\section{Coimmunoprecipitation}

Coimmunoprecipitation of ATP6AP2 and targeted proteins of the CLR-M fraction was performed according to $\mathrm{Lu}$ et al. [29]. Anti-ATP6AP2 or anti-caveolin or anti-

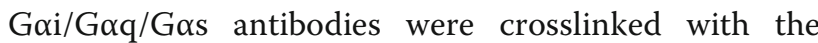
magnetic beads and then incubated for $30 \mathrm{~min}$ with the CLR-M proteins. After collection, wash and elution of the beads, immunoblotting analysis was realized on the immunoprecipitated (IP) samples to identify the binding partner. 


\section{Intracellular calcium assay}

Free intracellular calcium concentration $\left(\left[\mathrm{Ca}^{2+}\right]_{\mathrm{i}}\right)$ was determined using fura-2/AM as a calcium chelator. After serum-starving for $2 \mathrm{~h}, 10^{5}$ cells/ml were collected in BSA-KRH buffer and labeled with $160 \mathrm{nM}$ fura2/AM for $45 \mathrm{~min}$ at $37{ }^{\circ} \mathrm{C}$ under $5 \% \mathrm{CO}_{2}$. Then, cells were pelleted at $300 \times g$ for $5 \mathrm{~min}$, suspended in $\mathrm{KRH}$ buffer. After $15 \mathrm{~min}, \mathrm{CaCl}_{2}(1.5 \mathrm{mM})$ was added to the sample and kept with the cells for $15 \mathrm{~min}$. The total time elapsed between the end of the labeling and the first fluorescence determination was 35 min to allow full hydrolysis of the intracellular fura-2/AM ester. Fluorescence $(F)$ was determined in a Perkin-Elmer LS-5 spectrofluorimeter (excitation 340-450 nm, emission $510 \mathrm{~nm}$ ). All determinations were performed at $37{ }^{\circ} \mathrm{C}$. $\left[\mathrm{Ca}^{2+}\right]_{\mathrm{i}}$ was calculated using the following equation:

$$
\left[\mathrm{Ca}^{2+}\right]=K_{\mathrm{d}} \times\left[\left(R-R_{\min }\right) /\left(R_{\max }-R\right)\right] \times \beta,
$$

where $R$ is the ratio of fluorescence of the sample at 340 and $380 \mathrm{~nm}\left(F_{340} / F_{380}\right) ; R_{\max }$ and $R_{\min }$ represent the ratios for fura-2/AM at the same wavelengths of saturating $\mathrm{Ca}^{2+}$ determined by adding $1 \mu \mathrm{M}$ ionomycin and minimal $\mathrm{Ca}^{2+}$ determined by adding $15 \mathrm{mM}$ EGTA, respectively; $\beta$ is the ratio of fluorescence of fura-2/AM at $380 \mathrm{~nm}$ in minimal and saturating $\mathrm{Ca}^{2+}\left(F_{380 \mathrm{~min}} / F_{380}\right.$ $\max )$; and $K_{\mathrm{d}}$ is the dissociation constant of fura-2/AM for $\mathrm{Ca}^{2+}$, assumed to be $224 \mathrm{nM}$ at $37{ }^{\circ} \mathrm{C}$. All measures were determined by deducing the autofluorescence for each sample. Results were expressed as the concentration of intracellular calcium (nM).

\section{Statistical analysis}

Results are presented as the mean \pm SEM of four independent experiments performed in duplicate and analyzed for statistical significance (on absolute values) using Student's $t$ test. For all statistical tests, $P$ values were two-tailed and the level of significance was set at 0.05 .

\section{Results}

\section{Expression of ATP6AP2 in hPBMCs and hADSCs}

We were interested to evaluate the expression of ATP6AP2 for the first time in primary undifferentiated hADSCs in comparison to circulated hPBMCs isolated from the peripheral blood of the same donor. We assessed whether ATP6AP2 mRNA expression may vary between healthy and ND subjects. Subjects considered for neurological disorders were designated the neurodegenerative diseases (ND) group, divided into four subgroups as described in Methods. All of the ND groups and subgroups were sex and age matched with the healthy ones. First, the expression of ATP6AP2 mRNA was evaluated in undifferentiated cells: ATP6AP2 mRNA was significantly reduced in hADSCs and hPBMCs derided from the ND group (Fig. 1a-d) in comparison to the healthy group. Positive correlations were found between ADSC-expressing ATP6AP2 and PBMCexpressing ATP6AP2 in healthy $(R=0.84, P<0.00001)$ and ND $(R=0.64, P<0.001)$ groups (Fig. 1e, $\mathrm{f})$, indicating that the assessment of ATP6AP2 in hPBMCs can be considered a marker when the collection of adipose tissue is difficult to apply to donors with advanced ages. The moderate levels of ATP6AP2 mRNA expression in ND were not correlated to low levels of isolated cells, whether ADSCs or PBMCs (Fig. 1g, h), nor with age (data not shown).

Thus, we investigated the potential of hADSCs to differentiate into neural lineages during 37 days as indicated in Methods and assessed the expression of ATP6AP2 during this Neu-Dif: ATP6Ap2 mRNA and protein reached maximum levels of expression at day 30 of the differentiation; significantly lower levels of expressions were observed in the ND group (Fig. 1j, k). The decrease in ATP6AP2 protein in ND led us to investigate whether this decrement is due to an increase in the activity of the furin, the enzyme that cleaves $P R R$ intracellularly in the trans-Golgi to generate soluble prorenin receptor $(\mathrm{s}(\mathrm{P}) \mathrm{RR})$, which would subsequently be secreted by the cell. The quantification of $s(P) R R$ in the culture media showed no significant variations between healthy and ND subjects, indicating that the observed decreases in cellular proteins could be predominantly related to the transcriptional activity and not to an elevated furin activity, and indicate that undifferentiated cells had elevated furin activity (Fig. 11).

Analysis between subgroups within the ND population did not alter the represented results: in fact, all of the obtained data were similar and no significant variations were observed between the dual subgroups $(A D+P D)$ or $(\mathrm{ALS}+\mathrm{MS})$ and the controls.

\section{Neurogenic potential of hADSCs}

Cell morphology changes from a fusiform fibroblast type (MSC) to a neurocyte-like cell shaped in neurospheres (NSP) and getting a neural-like cell (NLC) at the end of ADSC differentiation (Fig. 2a). Similar morphologies were observed between cells derived from healthy and ND subjects.

The immunophenotyping of the cells was assessed at days $<0,30$ and 37 of the Neu-Dif by flow cytometry; we realized multiplex labeling of the single-cell suspension of the same sample using different fluorochromeconjugated antibodies. A switch from the mesenchymal phenotype $\left(\mathrm{CD} 34^{-} / \mathrm{CD} 45^{-} / \mathrm{CD} 3^{+} / \mathrm{CD}^{+} 0^{+} / \mathrm{CD} 105^{+}\right)$in undifferentiated MSCs to almost its absence (CD34- 
$\left.\mathrm{CD} 45^{-} / \mathrm{CD}^{-} 3^{-} / \mathrm{CD} 90^{-} / \mathrm{CD} 105^{-}\right)$indicated a significant cell differentiation; the apoptotic index indicated that there were no significant intragroup and intergroup variations between MSCs, NSPs and NLCs (Table 1).

To evaluate the neurogenic fate, the expression of neurogenic markers (TUJ1 and NeuN for neurons, O4 for oligodendrocytes, GFAP for astrocytes and nestin for undifferentiated MSCs) was evaluated by flow cytometry. First, the evaluation was realized on a mixed population without any purification. Double and triple labeling of the single-cell suspension of the same sample was realized each time to determine the degree of heterogeneity in the cell types present in the cultured samples. All of the analyzed data from the double (TUJ1-O4, TUJ1GFAP, NeuN-O4, NeuN-GFAP, TUJ1-NeuN) or triple (NeuN-O4-GFAP, TUJ1-O4-GFAP, TUJ1-NeuN-O4, TUJ1, NeuN, GFAP) labeling confirmed the results presented in Table 1: we observed marked increases in the expression of NeuN, TUJ1 and O4 at day 30 and day 37, with a concomitant decrement in the expression levels of GFAP and nestin, indicating predominant neuronal and oligodendrocyte phenotypes but an inhibition of astrocyte differentiation. Significant lower potential of Neu-Dif was shown in ND subjects. These results were confirmed by the purification assays: we purified separately $\mathrm{TUJ}^{(+)}$cells from $\mathrm{O}^{(+)}$cells as well as from GFAP $^{(+)}$cells of MSC $($day $<0)$ and NLC (day 37) populations (Table 2). The population of $\mathrm{TUJ}^{(1)}$ was largely expressed in NLCs in comparison to $4^{(+)}$and $\mathrm{GFAP}^{(+)}$, where TUJ1 ${ }^{(+)}$was increased markedly $(\sim 76 \%)$ with a concomitant moderate increase of $\mathrm{O}^{(+)}(\sim 21 \%)$ contrary to $\mathrm{GFAP}^{(+)}$which decreased in NLCs $(<1 \%)$, particularly of the healthy group. Interestingly, in comparison to the healthy group, the ND group showed significant differences in terms of a lower percentage of cells expressing $\mathrm{TUJ}^{(+)}(\sim 31 \%)$ and $\mathrm{O}^{(+)}(\sim 10 \%)$, contrary to the higher percentage of $\mathrm{GFAP}^{(+)}$cells $(\sim 8 \%)$. Importantly, the expression of ATP6AP2 in these purified cell populations showed significant variation between healthy and ND-derived cells only in the case of $\mathrm{TUJ} 1^{(+)}$population, the differentiated neuronal-like cells (18-fold vs 6 -fold increases at day 37 , healthy vs ND, respectively) (Table 2).

MSCs and NLCs (at day $<0$ and day 37, respectively) derived from healthy hADSCs were then stained with the neuronal marker TUJ1 and evaluated by confocal microscopy: we remarked an absence of TUJ1 in MSCs contrary to its high detection in differentiated NLCs (Fig. 2b). The heterogeneity of the differentiated population was confirmed by triple labeling of the single-cell suspension of the differentiated hADSCs at day 37 by staining TUJ1/O4/GFAP (Fig. 2c); it is important to note that the GFAP-labeled cells were nearly absent from this population.

\section{ATP6AP2 regulates $p$-ERK during neural differentiation}

ERK had been confirmed as a key mediator of the ATP6AP2 signaling and can be activated by hRenin $[5,30]$. Then, we checked the expression levels of total and phosphorylated ERK (p-ERK) proteins during neural differentiation. We observed (Fig. 3a) that total ERKs did not vary significantly between healthy and ND groups but p-ERKs increased. hRenin induced significant p-ERK particularly in healthy NLCs (Fig. 3b). These effects were abolished when cells were treated with PD98059 $(10 \mu \mathrm{M})$, which is a selective inhibitor of MEK1/2 (the direct upstream kinase activator of ERK1/2) that inhibits ERK1/2 phosphorylation (Fig. 3b), indicating that ERK is required for the ATP6AP2 action.

\section{Involvement of ATP6AP2 in Wnt signaling responsiveness in NLCs}

Wnt signal pathways play key roles in neural differentiation [31]. To assess the Wnt signaling responsiveness, differentiated AD-NLCs were treated with canonical Wnt agonist (Wnt3a)/Wnt antagonist (Dkk-1) or noncanonical ligand (Wnt5a) for $24 \mathrm{~h}$ before adding hRenin as previously: $\mathrm{p}$-JNK and translocation of $\beta$-catenin into the nucleus was evaluated. In fact, Wnt5A had been reported to activate Wnt/PCP signaling, which leads to the phosphorylation of JNK and its downstream target c-Jun $[32,33]$, whereas Wnt3A activates Wnt/B-catenin signaling by inducing its translocation into the nucleus [34]. Our results indicated in differentiated AD-NLCs a noncanonical Wnt signaling responsiveness involving ATP6AP2, not canonical signaling (Fig. 4).

First, hRenin induced efficiently p-JNK, but significant lower levels were seen in ND (Fig. 4a, b); total JNK did not vary significantly. This induction of phosphorylation has been enhanced strongly by Wnt5a; however, Wnt3a and Dkk1 failed to enhance the hRenin-induced JNK responsiveness (Fig. 4c, d). Second, hRenin failed to significantly induce $\beta$-catenin translocation into the nucleus (Fig. 4a, b); In fact, it was very difficult to detect the $\beta$-catenin protein in the nucleus or in the cytoplasm. The identification of the very modest levels in the nucleus allowed us to distinguish that there were no significant variations (Fig. 4e, f).

\section{Induction of ATP6Ap2 is accompanied with a transition from $W n t / \beta$-catenin to Wnt/PCP signaling, and from CAV to FLOT}

The very low expression levels of $\beta$-catenin in differentiated NLCs led us to check its expression in undifferentiated cells. Therefore, the sequential activity of Wnt/ $\beta$ catenin and Wnt/PCP signaling was examined during neural differentiation of ADSCs. Our results showed an 
a

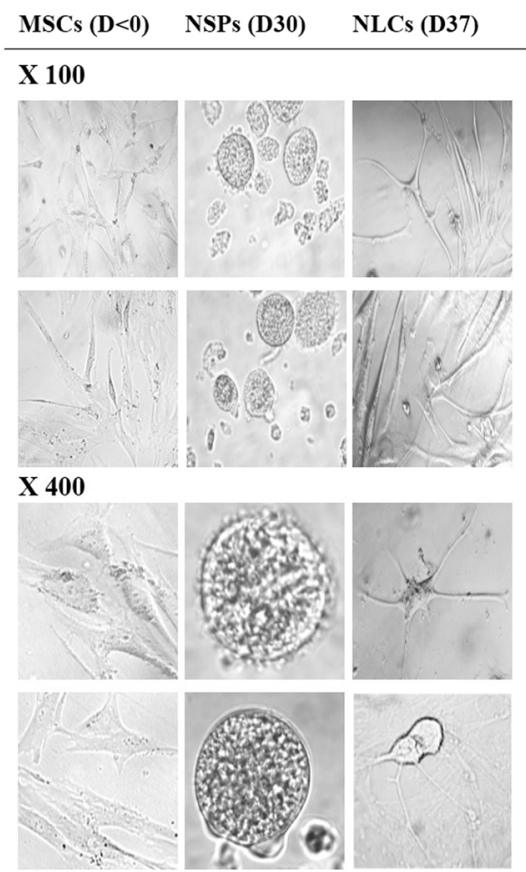

c
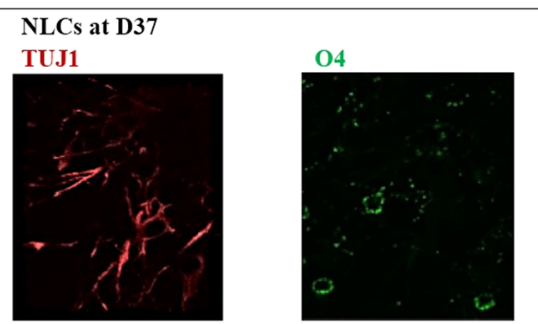

b

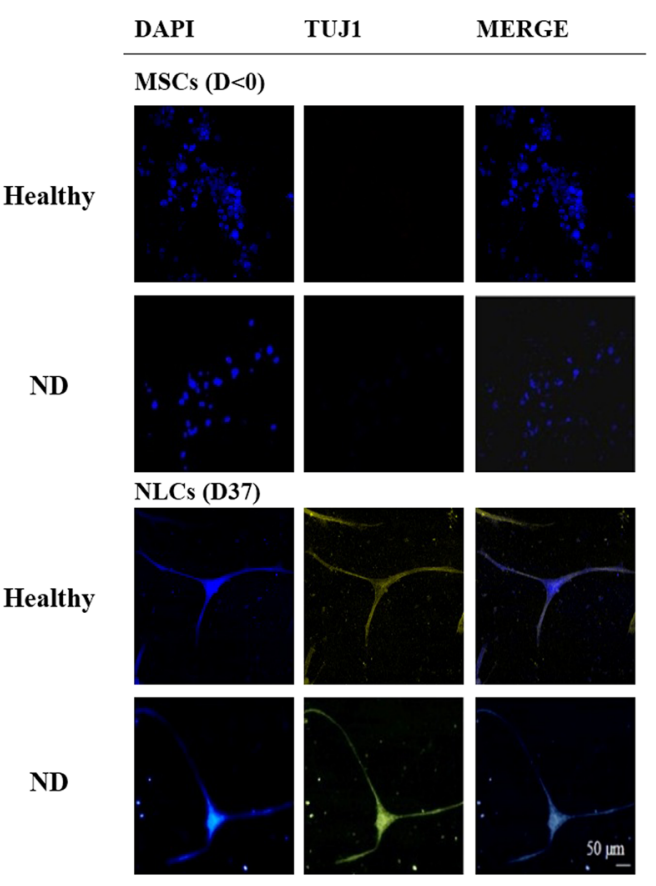

b

$\operatorname{MSCs}(\mathrm{D}<0)$

\section{.}
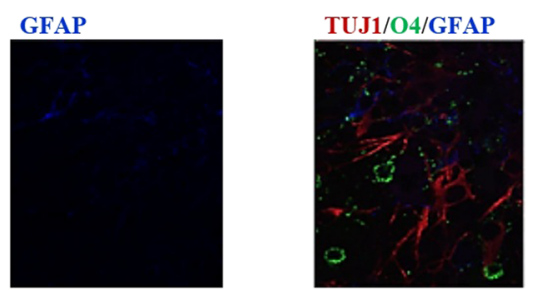

Fig. 2 Morphological cell changes during Neu-Dif. hADSCs isolated from healthy or ND subjects and differentiated for 37 days as indicated in Methods. Cell morphology at days 0 (MSCs), 21 (NSPs) and 37 (NLCs) of neural differentiation showing progeny of single cell-derived neurosphere and neural-like structures (a). Immunostaining assays of MSCs (at day <0) and NLCS (at day 37) stained with TUJ1 (yellow) and nuclei counterstained with DAPI (blue) (b). Triple labeling of the single-cell suspension fully differentiated at D37 stained with TUJ1 (red), O4 (green) and GFAP (blue) (c). D day, DAPI 4',6-diamidino-2-phenylindole, MSC mesenchymal stem cell, ND neurodegenerative disease, NLC neuron-like cell, NSP neurosphere-like structure

attenuation of the Wnt/ $/$-catenin pathway during neural differentiation whereas Wnt/PCP was unregulated. Upon differentiation, expression levels of canonical components such as $\beta$-catenin, LRP-5 and LRP-6 were gradually and significantly downregulated (Fig. 5a). Concurrently, the major noncanonical Wnt/PCP components such as Fzd-6, PTK-7 and VANGL (Fig. 5b) and the core PCP genes Celsr1/2/3 were continually and significantly upregulated (Fig. 5c). Simultaneously, Wnt-5a and Wnt-7a were markedly and significantly increased, contrary to Wnt-3a which was downregulated (Fig. 5d). No significant changes were noted in the mRNAs levels of Fzd-3, Wnt-4a and Wnt-11.

The attenuation of Wnt/ $\beta$-catenin signaling could be related to a modulation in the expression of CAV, a major component of the plasma membrane microdomains where the Wnt signaling cascade can be initiated. In fact, it has been reported that CAV-1 plays a crucial role in inhibiting neuronal and oligodendroglia differentiation of neural stem cells in vitro through inhibition of $\beta$-catenin expression [35] and via a VEGF signaling pathway [14]. In addition, VEGF and ERK have been considered previously as downstream effectors of ATP6AP2 in endothelial cells, and treatment with hProrenin increases mRNA expression of VEGF and phosphorylation of ERK [11]. Thus, we determined the expression of $\mathrm{CAV}-1 / 2 / 3$ as well as VEGF. We observed that $\mathrm{CAV}-1 / 2 / 3$ were highly expressed in undifferentiated ADSCs and their mRNA expressions were dramatically attenuated during neural differentiation (Fig. 5e). CAV-1 was the most expressed isoform of CAV, whereas VEGF mRNA 
Table 1 Immunophenotyping, apoptotic index and distribution of neurogenic markers on unpurified mixed population

\begin{tabular}{|c|c|c|c|}
\hline & $\begin{array}{l}\text { MSCs } \\
\text { Day <0 }\end{array}$ & $\begin{array}{l}\text { NSPs } \\
\text { Day } 30\end{array}$ & $\begin{array}{l}\text { NLCs } \\
\text { Day } 37\end{array}$ \\
\hline \multicolumn{4}{|c|}{ Differentiation of hADSCs in healthy subjects } \\
\hline $\mathrm{CD}_{3} 4^{(+)}$ & $1.26 \pm 2.01$ & $0.18 \pm 1.64$ & $0.51 \pm 2.60$ \\
\hline $\mathrm{CD} 45^{(+)}$ & $1.28 \pm 1.17$ & $0.71 \pm 0.92$ & $0.07 \pm 1.34$ \\
\hline $\mathrm{CD} 3^{(+)}$ & $90.12 \pm 5.52$ & $24.51 \pm 10.34^{*}$ & $6.77 \pm 3.68^{* *}$ \\
\hline $\mathrm{CD} 0^{(+)}$ & $95.59 \pm 6.80$ & $4.07 \pm 3.76^{* *}$ & $1.94 \pm 2.1^{* * *}$ \\
\hline $\mathrm{CD} 105^{(+)}$ & $84.33 \pm 5.33$ & $18.68 \pm 4.51^{*}$ & $1.26 \pm 1.90^{* * *}$ \\
\hline Annexin $\mathrm{V}^{(-)} / \mathrm{Pl}^{(-)} / 7 \mathrm{AAD}^{(-)}$(viable cells) & $98.97 \pm 0.71$ & $96.20 \pm 1.25$ & $96.85 \pm 0.83$ \\
\hline Annexin $\mathrm{V}^{(+)} / \mathrm{PI}^{(-)} / 7 \mathrm{AAD}{ }^{(-)}$(early-apoptotic cells) & $0.76 \pm 0.90$ & $2.11 \pm 1.01$ & $2.03 \pm 1.04$ \\
\hline Annexin $\mathrm{V}^{(+)} / \mathrm{PI}^{(+)} / 7 \mathrm{AAD}{ }^{(+)}$(late apoptotic, necrotic cells) & $0.27 \pm 0.71$ & $1.69 \pm 0.99$ & $1.12 \pm 1.10$ \\
\hline NeuN & $1.62 \pm 1.54$ & $58.14 \pm 3.32^{* *}$ & $78.12 \pm 6.28^{* *}$ \\
\hline TUJ1 & $4.49 \pm 1.62$ & $60.48 \pm 5.25^{* *}$ & $89.09 \pm 8.67^{* *}$ \\
\hline $\mathrm{O} 4$ & $1.28 \pm 2.40$ & $28.16 \pm 4.11^{*}$ & $26.33 \pm 3.29^{*}$ \\
\hline GFAP & $24.14 \pm 2.58$ & $4.26 \pm 1.84^{* *}$ & $1.20 \pm 1.24$ ** \\
\hline Nestin & $54.01 \pm 8.41$ & $20.15 \pm 2.64^{*}$ & $0.81 \pm 2.62^{* *}$ \\
\hline \multicolumn{4}{|c|}{ Differentiation of hADSCs in ND subjects } \\
\hline $\mathrm{CD}_{3} 4^{(+)}$ & $1.82 \pm 1.72$ & $0.08 \pm 1.21$ & $0.71 \pm 1.05$ \\
\hline $\mathrm{CD} 45^{(+)}$ & $3.69 \pm 1.88$ & $0.90 \pm 1.14$ & $0.11 \pm 1.92$ \\
\hline $\mathrm{CD} 3^{(+)}$ & $79.49 \pm 3.42$ & $4.06 \pm 2.66^{* *}$ & $2.90 \pm 2.03^{* * *}$ \\
\hline $\mathrm{CD}^{(+)}$ & $93.51 \pm 3.01$ & $0.73 \pm 1.40^{* * *}$ & $0.98 \pm 1.74^{* * *}$ \\
\hline CD105 ${ }^{(+)}$ & $86.28 \pm 6.76$ & $13.53 \pm 5.18^{* *}$ & $5.04 \pm 7.18^{* *}$ \\
\hline Annexin $\mathrm{V}^{(-)} / \mathrm{PI}^{(-)} / 7 \mathrm{AAD}^{(-)}$(viable cells) & $97.12 \pm 1.13$ & $93.10 \pm 2.44$ & $98.33 \pm 2.91$ \\
\hline Annexin $\mathrm{V}^{(+)} / \mathrm{PI}^{(-)} / 7 \mathrm{AAD} \mathrm{D}^{(-)}$(early apoptotic cells) & $1.96 \pm 0.76$ & $4.71 \pm 1.82$ & $0.02 \pm 2.16$ \\
\hline Annexin $\mathrm{V}^{(+)} / \mathrm{PI}^{(+)} / 7 \mathrm{AAD}^{(+)}$(late apoptotic, necrotic cells) & $0.92 \pm 1.33$ & $2.19 \pm 1.37$ & $1.65 \pm 0.24$ \\
\hline NeuN & $0.37 \pm 1.09$ & $45.20 \pm 2.07^{* *}$ & $50.31 \pm 2.53^{* *, \S}$ \\
\hline TUJ1 & $1.94 \pm 1.13$ & $40.11 \pm 4.17^{* *}, \S$ & $52.65 \pm 6.24 * *$, §§ \\
\hline $\mathrm{O} 4$ & $4.13 \pm 5.10$ & $38.14 \pm 5.23^{* *}$ & $18.04 \pm 1.88^{*}, \S$ \\
\hline GFAP & $34.05 \pm 3.67^{\S}$ & $4.19 \pm 2.12^{* *}$ & $2.43 \pm 4.16^{* *}$ \\
\hline Nestin & $73.56 \pm 7.82^{\S}$ & $16.20 \pm 3.75^{* *}$ & $1.05 \pm 2.35^{* *}$ \\
\hline
\end{tabular}

Cells were labeled with fluorescence-coupled antibodies against CD34, CD45, CD73, CD90, CD105, Annexin V, 7AAD, propidium iodide solution (PI) and neural markers and analyzed using a MACSQuant flow analyzer as indicated in Methods. Results presented as mean \pm SEM and presented as the percentage of cell surface marker per cell type (\% of total cells) of all subjects each performed in duplicate in each group. Cells collected at day 0 before the induction of the differentiation (MSCs) and days 30 and 37 of differentiation (NSPs and NLCs, respectively)

hADSC human adipose-derived mesenchymal stem cell, MSC mesenchymal stem cell, ND neurodegenerative disease, NLC neural-like cell, NSP neurosphere-like structure

${ }^{*} P<0.05,{ }^{* *} P<0.01$ and ${ }^{* * * *} P<0.005$ : NSPs/NLCs vs MSCs

${ }^{\S} P<0.05,{ }^{\S \S} P<0.01$ : ND vs healthy

expression increased during neural differentiation contrary to its antagonist PEDF (Fig. 5f). These results are concomitant with those of $\beta$-catenin and the observed increases in p-ERK, and confirm the potential negative role of CAV-1 in inhibiting Neu-Dif.

However, the significant lack of CAV-1 in AD-NLCs suggests that other markers of lipid raft microdomains might be essential to the late phase of the neural differentiation. Among these components, FLOT belongs to a larger class of markers expressed in cells that lack CAV (e.g. neurons) [20], suggesting that it may represent a functional analogue of CAV-negative cells. As expected, the expression of FLOT-1 and FLOT-2 mRNAs increased during neural differentiation (Fig. 5g).

The profile of results obtained for the ND group was similar to that of healthy subjects but with moderate variations (data not shown), except CAVs which were not completely attenuated during neural differentiation (Fig. 5h).

\section{Subcellular distribution of ATP6Ap2 protein}

The transition of expression from CAV to FLOT in ADNLCs led us to check whether the subcellular distribution 
Table 2 Distribution of $\mathrm{TUJ}^{+} / \mathrm{O}^{+} / \mathrm{GFAP}^{+}$purified cells before and after hADSC differentiation, and their relative mRNA ATP6AP2 expression

\begin{tabular}{|c|c|c|c|}
\hline & \multicolumn{2}{|c|}{$\begin{array}{l}\text { Immunophenotyping } \\
\text { (\% of total cells) }\end{array}$} & \multirow{2}{*}{$\begin{array}{l}\text { ATP6AP2 mRNA } \\
\text { (fold expression relative to MSCs) } \\
\text { NLCs } \\
\text { Day } 37\end{array}$} \\
\hline & $\begin{array}{l}\text { MSCs } \\
\text { Day <0 }\end{array}$ & $\begin{array}{l}\text { NLCs } \\
\text { Day } 37\end{array}$ & \\
\hline \multicolumn{4}{|l|}{ Healthy } \\
\hline TUJ1 $1^{(+)}$ & $2.87 \pm 0.14$ & $76.04 \pm 0.22^{* * *}$ & $18.2 \pm 0.49^{* * *}$ \\
\hline $\mathrm{O} 4^{(+)}$ & $5.10 \pm 0.38$ & $21.12 \pm 0.53^{* *}$ & $4.27 \pm 0.80^{*}$ \\
\hline $\mathrm{GFAP}^{(+)}$ & $20.92 \pm 0.46$ & $0.91 \pm 0.65^{* * *}$ & $1.31 \pm 0.42$ \\
\hline \multicolumn{4}{|l|}{ ND } \\
\hline TUJ1 $1^{(+)}$ & $1.94 \pm 0.90$ & $31.44 \pm 0.95^{* *}, \S \S$ & $6.11 \pm 0.83^{*}, \S \S$ \\
\hline $\mathrm{O} 4^{(+)}$ & $1.08 \pm 0.67$ & $10.08 \pm 0.48^{*}, \S$ & $5.09 \pm 0.78^{*}$ \\
\hline $\mathrm{GFAP}^{(+)}$ & $32.16 \pm 0.71^{\S}$ & $8.23 \pm 0.51^{*}, \S$ & $1.07 \pm 0.95$ \\
\hline
\end{tabular}

hADSC human adipose-derived mesenchymal stem cell, MSC mesenchymal stem cell, ND neurodegenerative disease, NLC neural-like cell, NSP neurosphere-like structure

${ }^{a} 2 \times 10^{7}$ cells derived each from healthy or ND subjects were applied to purification at day $<0$ and day 37 as indicated in Methods. Three positive fractions were purified separately: TUJ1 ${ }^{(+)}, \mathrm{O}^{(+)}$and GFAP(+) cells. Cells were labeled with anti-TUJ1-FITC or anti-O4-APC or anti-GFAP-PE and analyzed using a MACSQuant flow analyzer as indicated in Methods. Results presented as mean \pm SEM of two independent experiments each performed in duplicate

${ }^{\mathrm{b}}$ Expression of ATP6AP2 mRNA was determined by RT-qPCR on the purified fraction. Results expressed as fold variation relative to MSCs after normalization to GAPDH. Cells were collected at day 0 before the induction of the differentiation (MSCs) or at day 37 of the differentiation (NLCs)

${ }^{*} \mathrm{P}<0.05,{ }^{* * *} \mathrm{P}<0.01$ and ${ }^{* * * *} \mathrm{P}<0.005$ : NLCs vs MSCs

${ }^{\S} P<0.05,{ }^{\S \varsigma} P<0.01$ : ND vs healthy

of ATP6AP2 could be affected. Cells were subjected to isolation of detergent-resistant microdomains (CLR-Ms).

The data demonstrated that ATP6AP2 is mainly localized intracellularly (Fig. 6a). Cell fractionation with various organelle markers showed colocalization of ATP6AP2 with the ER marker (TRAP $)$ but little colocalization was observed with the Golgi (TGN38, GM130), endosomal (EEA1) and lysosomal (LAMP1) markers and total absence with the nuclear (Nucleoporin, Lamin A) markers (Fig. 6b), indicating that ATP6AP2 is indeed highly concentrated in a mixed membrane compartment including $\mathrm{ER}$ and
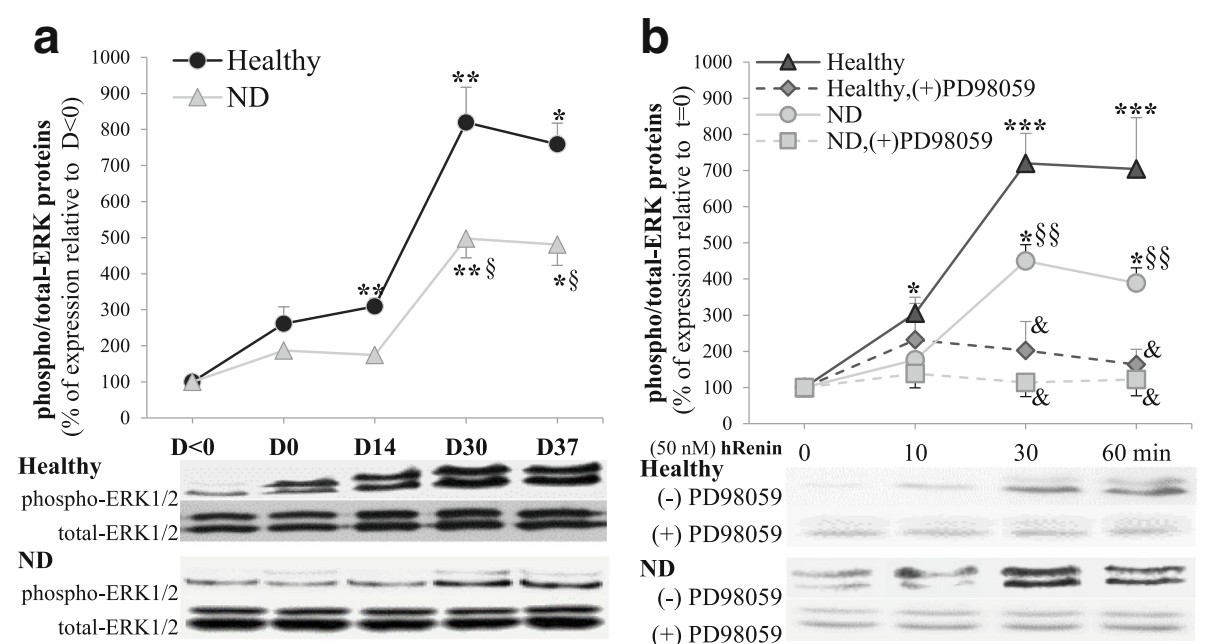

Fig. 3 ATP6AP2 signaling is dependent on MAPK in hADSCs. hADSCs isolated from healthy and ND subjects and differentiated for 37 days. Cell lysates (80-150 $\mathrm{\mu g}$ of protein) separated by SDS-PAGE and immunoblotted with antibodies raised against p-ERK and total ERK. Proteins expression determined during differentiation from day <0 to day 37 (a) or in NLCs at day 37 to evaluate the renin responsiveness (b). NLCs at day 37 treated after serum starvation overnight with human recombinant renin (hRenin, 50 nM) for indicated time course with or without 30 M PD98059; to prevent any ERK phosphorylation potentially due to ANG II generation, Losartan $(10 \mu \mathrm{M})$ and PD123319 (10 $\mu \mathrm{M})$ ANG II blockers added during time course; quantitative variations in total and p-ERK amounts of p42 and p44 combined and shown. ${ }^{\star \S} P<0.05$, ${ }^{*}{ }^{\S \&} p<0.01$, ${ }^{* * *} p<0.005$ : ${ }^{*} \mathrm{D} 0 /$ $14 / 30 / 37$ vs day $<0$ or hRenin T10/30/60 vs $T=0,{ }^{\S}$ ND vs healthy, ${ }^{\&}$ PD 98059 -treated vs untreated cells. D day, ERK extracellular signal-regulated kinase, ND neurodegenerative disease, $T$ time 
a

Healthy

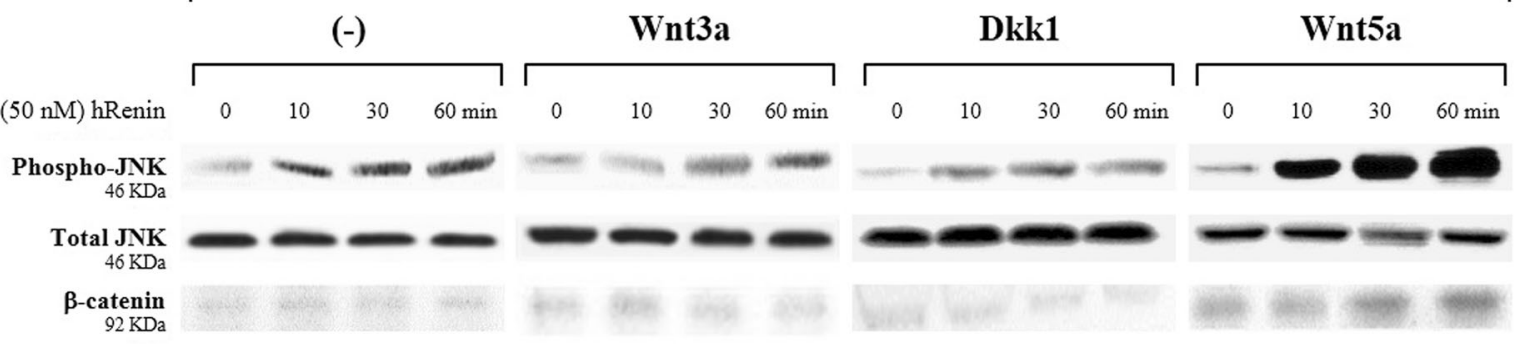

b

ND
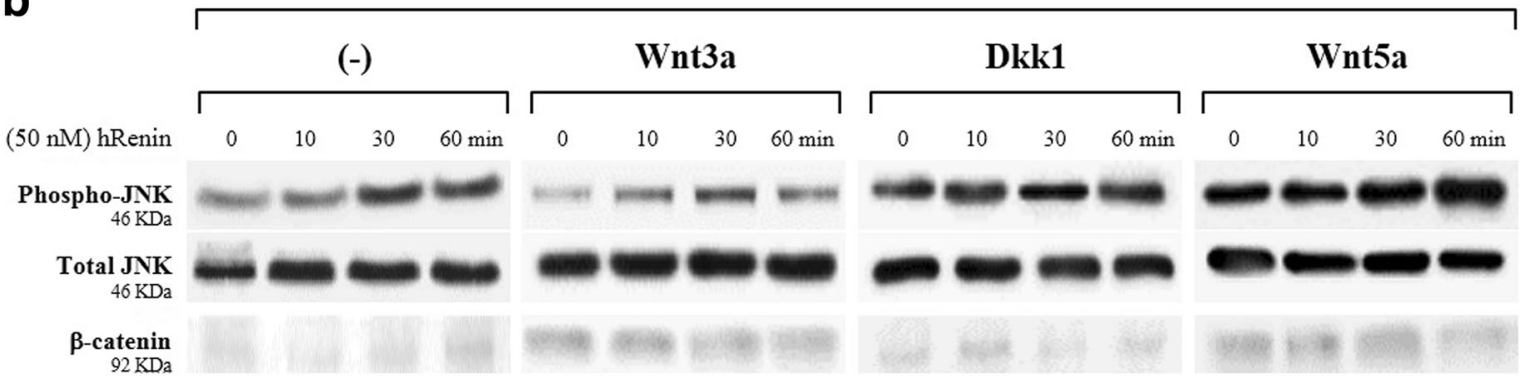

C
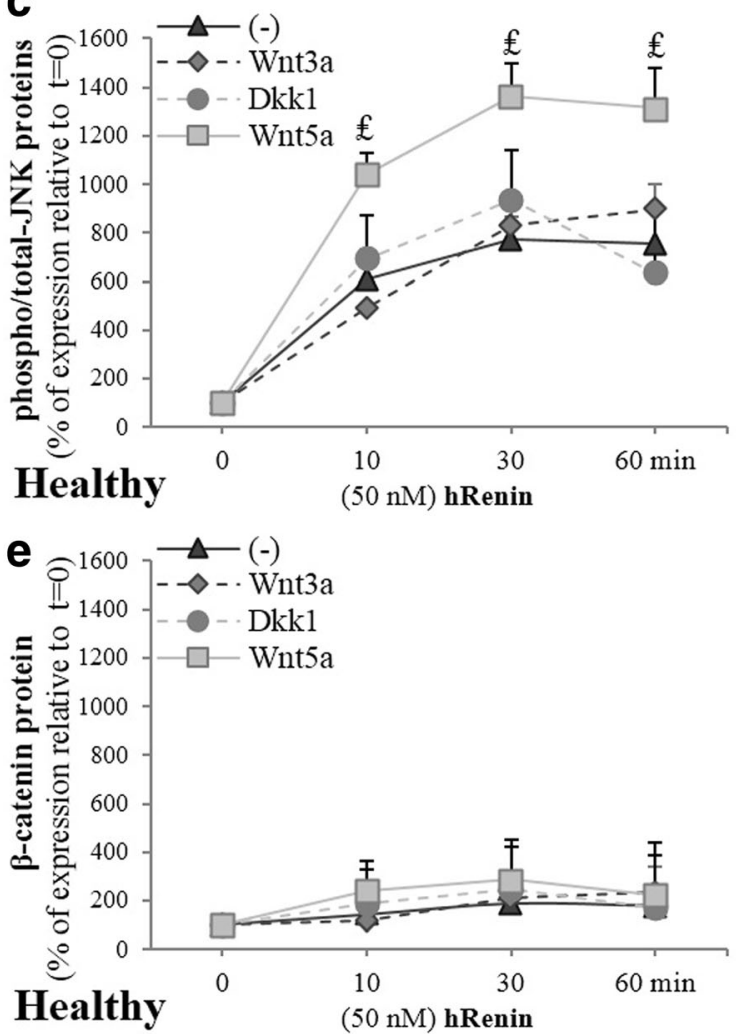

d
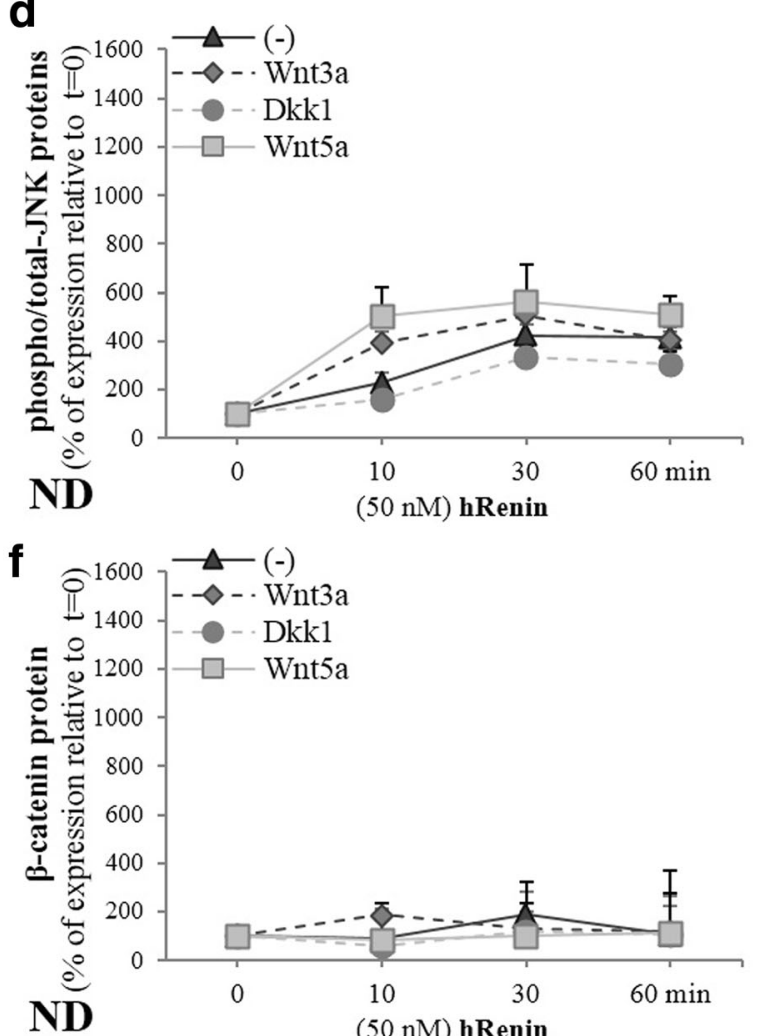

Fig. 4 ATP6AP2 signaling is dependent on Wnt(s) signaling in hADSCs. hADSCs isolated from healthy and ND subjects and differentiated for 37 days. Cell lysates (80-150 $\mu \mathrm{g}$ of protein) separated by SDS-PAGE and immunoblotted with antibodies raised against p-JNK, total JNK1 and $\beta$-catenin. Role of renin and canonical/noncanonical Wnt signaling evaluated. NLCs at day 37 treated after serum starvation overnight with human recombinant renin (hRenin, $50 \mathrm{nM}$ ) as indicated in Fig. 3. NLCs at day 36 treated overnight with Wnt3a (50 ng/ml, 24 h) or Wnt-antagonist DKK1 $(200 \mathrm{ng} / \mathrm{ml}, 24 \mathrm{~h})$ and Wnt5a (100 ng/ml, 24 h), and at day 37 with hRenin (50 nM, 0-1 h). Representative blots (a, b) and quantitative variations (c-f) shown. ${ }^{£} P<0.01$, Wnt5a-treated vs untreated cells. hRenin human recombinant renin, JNK Jun N-terminal kinase 
endosomes. Interestingly, parallel increases were seen also in the plasma membrane (Fig. 6c), particularly in CLR-Ms. In ND-derived cells, similar distribution was observed with lower levels of expression (Fig. 6d-f). The increases were accompanied with a significant drop of CAV at the cellular level, more importantly from PM and CLR-Ms (Fig. 6g), with strong increases of FLOT in CLR-Ms (Fig. 6h). Comparable subcellular distribution of CAV and FLOT was also observed in ND-derived cells (data not shown).

\section{GW4869 (GW) inhibits ATP6AP2, suspends its accumulation in CLR-Ms and reduces exosome release} CLR-Ms are plasmalemmal microdomains enriched in scaffolding proteins, but also in sphingolipids and cholesterol. We hypothesized that any disruption of these microdomains may affect ATP6AP2. Pharmacological treatments were used to achieve this goal targeting the mass of cholesterol or sphingolipids in CLR-Ms. First, we tried to deplete cholesterol by treating AD-MSCs with the most commonly used methyl- $\beta$-cyclodextrin (MBCD), a specific cholesterol-binding agent. MBCD (0-100 mM) was added for $48 \mathrm{~h}$ to adherent cells at day -2 . Unfortunately, we did not succeed in maintaining the cells in adherence and viable along the whole process of differentiation. With doses superior or equal to $100 \mathrm{nM}$, the cytotoxicity effects and the apoptotic index were observed from the 4th day of differentiation depending on the utilized dose. Inferior to this dose, it was impossible to decrease significantly the cholesterol levels from CLR-Ms. Other cholesterol sequestering agents were used, nystatin and filipin: the disruption of CLR-Ms was optimal but the same problem as that of MBCD occurred. Cholesterol biosynthesis inhibitors (AY 9944, Triparanol) led to a marked reduction in cholesterol content, although here it was impossible to achieve the complete neural differentiation (success up to 9-11 days). However, when analyzing the expression of ATP6AP2 during these days, we observed intracellular increase in the ATP6AP2 protein levels (data not shown).

Second, the other main components of CLR-Ms are sphingolipids (sphingomyelin and glycosphingolipid). Sphingomyelinases (SMase), ceramidases and glycosyl ceramide synthase (GCS) are key enzymes of sphingolipid metabolism that regulate the formation and degradation of ceramide. Inhibition of ceramidase (by D-e-MAPP, $10 \mu \mathrm{M}, 48 \mathrm{~h}($ day $-2 / 0)$ ) and GCS (by DL-1-PPMP, $20 \mu \mathrm{M}, 48 \mathrm{~h}($ day $-2 / 0))$ to increase the levels of ceramide had no cytotoxic effects and maintained cells in differentiation without significant loss (12-17\%), causing sequential CLR-M depletion of CAV and accumulation of FLOT with moderate positive regulation of ATP6AP2. Complete blockage of ceramide synthesis by inhibiting the serine palmitoyltransferase (by ISP-1, $2 \mu \mathrm{M}, 48 \mathrm{~h}($ day $-2 / 0)$ ) had opposite effects but not potently (data not shown). Therefore, we aimed to block the activity of SMase: gluthatione GSH treatment $(10 \mathrm{mM}, 48 \mathrm{~h}($ day $-2 / 0))$ by inhibiting $\mathrm{N}$ SMase had elevated cytotoxic effects starting at day 5-7 of the differentiation (viability ranged from 23 to $45 \%$ ); when ADSCs were treated with lower doses (order of micromolar and nanomolar), the viability of the cells started to be affected at day 11-12 (data not shown). Only the specific N-SMase inhibitor GW4869 (GW) treatment $(20 \mu \mathrm{M}$, $48 \mathrm{~h}($ day $-2 / 0)$ ) was efficient, without significant loss of cells (viability $>93 \%$, apoptotic index $<3.8 \%$ ), and achieving complete neural differentiation. GW induced significant accumulation of sphingomyelin in the plasma membrane, particularly in CLR-Ms with maximum effect at days 30 and 37. In the following section of this manuscript, we will present the data obtained in GWtreated ADSCs.

Our results showed that in GW-treated cells, neuronal TUJ1 and oligodendrocyte O4 markers were downregulated contrary to the astrocyte GFAP (Fig. 7a-c). Concurrently, considerable decreases in the levels of expression of ATP6AP2 protein (Fig. 7d-f) were shown particularly in CLR-Ms, but surprisingly accumulated in PNS, indicating a relocalization of ATP6AP2 in intracellular compartments. Thus, GW reversed successfully the expression of ATP6AP2. Disruption of CLR-Ms was reflected by a dynamic change in the abundance of CAV (Fig. $7 \mathrm{~g}-\mathrm{i}$ ) and FLOT (Fig. 7j-l). GW induced increases in the levels of cellular CAV, strongly in CLR-Ms and slightly in PNS. No significant changes were observed in the cellular levels of FLOTs, but rather decreases in CLR-Ms indicating efficiently a reversible effect. However, FLOT accumulated significantly in intracellular compartments, indicating the likely reason why ATP6AP2 was increased in PNS after treatment with GW.

Several reports have described release of exosomes from neural cells: differentiated [36] and developing neurons [37], oligodendrocytes [38] and astrocytes [39]. We hypothesized that in ATP6AP2-downregulated cells, the relocalization of ATP6AP2 concomitantly with FLOT, from the CLR-Ms into intracellular compartments, may be due to inhibition of exosome release. Several markers of the tetraspanin family including CD9, CD63 and CD81 are highly enriched in exosomal membranes; GW-pretreated cells significantly impaired release of exosomes during neural differentiation as observed by a significant reduction in the expression of CD9/CD63/CD81 (Fig. 8a), and the reduction of FLOT and CAV (Fig. 8b) recovered in the exosome fraction. These results collectively indicate that the disruption of CLR-Ms by GW affects exosome release and thus redistributes CLR-M components to intracellular sites. Finally, to know whether the Wnt signaling pathway can be affected, we investigated the effects of GW on the exosomal 

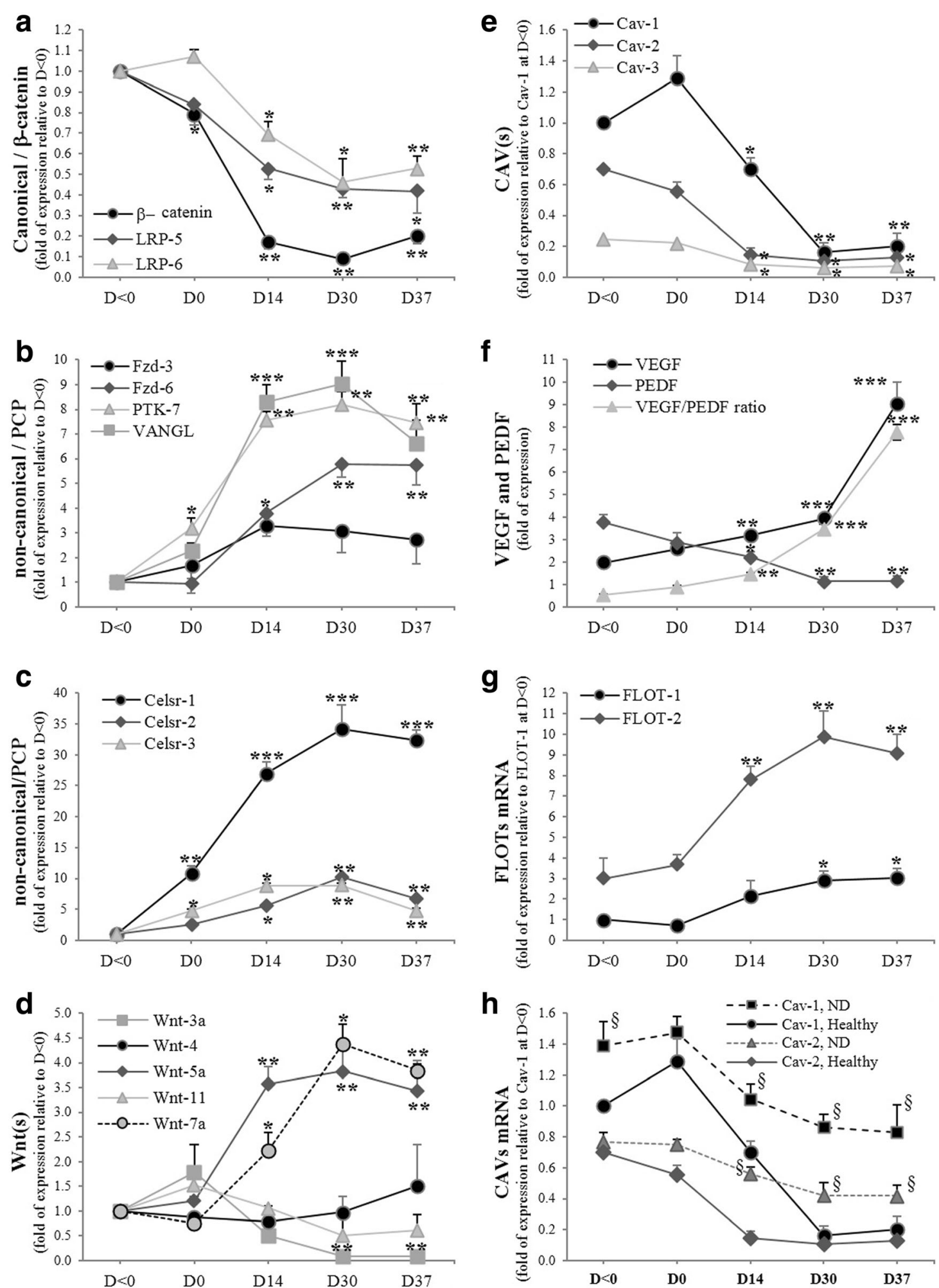

Fig. 5 Transition of Wnt signaling responsiveness from Wnt/B-catenin to Wnt/planar cell polarity (PCP) during Neu-Dif. hADSCs cultured and differentiated as indicated in Methods; mRNA expression determined by RT-qPCR and normalized to GAPDH. Expression levels of canonical and noncanonical genes (ß-catenin, LRP5 and LRP6 (a), Fzd-3, Fzd-6, PTK-7 and VANGL (b), Celsr(s) (c), Wnt(s) (d)), and caveolar/lipid raft key genes (CAVs (e), FLOTs (f) ) and target gene of ATP6AP2 (VEGF/PEDF (g)) for healthy group. CAV-1/CAV-2 expression in ND group (h). ${ }^{* \S} P<0.05$, ${ }^{* *} P<0.001$, ${ }^{* * *} P<0.0001:{ }^{*} D 0 / 14 /$ $30 / 37$ vs day $<0,{ }^{5} \mathrm{ND}$ vs healthy. CAV caveolin, D day, FLOT flotillin, PEDF pigment epithelium-derived factor, VEGF vascular endothelial growth factor 
export of $\beta$-catenin. Consistent with the reduction in exosome release, a marked reduction in the release of $\beta$ catenin was also observed (Fig. 8b), indicating an accumulation of $\beta$-catenin in the ATP6AP2-downregulated cell. In fact, during Neu-Dif, the levels of $\beta$-catenin mRNA increased moderately; however, the levels of its protein in CLRMs increased significantly (Fig. 8c). No significant variations of CD9/63/81 were observed in GW-treated cells during Neu-Dif (data not shown). These results indicate that GW blocks exosome release by sequestration of $\beta$ catenin in the plasma membrane and not due to an effect at the transcriptional level.

\section{Ga proteins crosslink ATP6AP2 to CAV}

To further evaluate the interaction between ATP6AP2 and CAV, we performed co-IP experiments on CLR-Ms. Unexpectedly, no direct interactions were found between ATP6AP2 and CAV in CLR-Ms (Fig. 9a). These results led us to check by co-IP experiments whether any potential crosslink could occur between these two

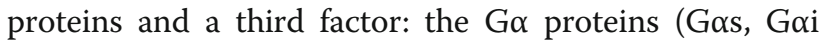

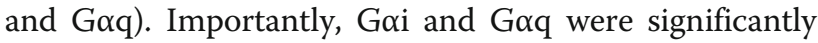
detected in CLR-Ms crosslinked with CAV and ATP6AP2 respectively, contrary to Gas which was unchanged. During differentiation of ADSCs, we observed that IP:ATP6AP2 retained strongly Goq in CLR-Ms of differentiated cells and moderately Goi in CLR-Ms of undifferentiated cells, with no interaction with Gas. However, IP:CAV retained strongly Goi in CLR-Ms of undifferentiated cells and these interactions disappeared completely from CRL-Ms of differentiated

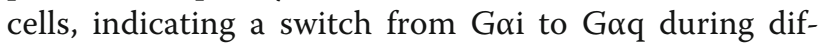
ferentiation (Fig. 9b). These concomitant increases of ATP6AP2 and Gaq were coupled to significant decreases of CAV and Goi, indicating the eventual mechanism by which ATP6AP2 acts.

To confirm the involvement of $\mathrm{G} \alpha$ proteins, we treated cells with the Gos activator cholera toxin (CTX), the Gaq protein inhibitor YM254890 (YM) and the Goi inhibitor pertussis toxin (PTX), and assessed the activation of the transcription factors CREB and c-Jun, major targets of ERK signaling in neuronal cells, and determined the exosome release. In control cells, the phosphorylation of CREB and c-Jun were induced during differentiation (Fig. 10). PTX increased significantly the nuclear $\mathrm{p}-\mathrm{CREB} / \mathrm{c}-\mathrm{Jun}$ and the release of exosomes, whereas YM and YM + PTX abolished their activities during differentiation. The involvement of Gaq led us to assess whether the enhanced release of exosomes was due to an increase in intracellular $\mathrm{Ca}^{2+}$. Expectedly, $\left[\mathrm{Ca}^{2+}\right]_{\mathrm{i}}$ increased significantly during NeuDif; a strong increase was shown with PTX, whereas a blockade was obtained in the case of YM and YM + PTX (Fig. 10a). In addition, TUJ1 protein increased strongly in PTX-treated cells and decreased significantly in YM/YM + PTX-treated cells, indicating a

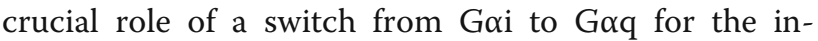
duction of Neu-Dif (Fig. 10a). No significant changes were observed with CTX.

To assess the involvement of ATP6AP2, NLCs at day 37 were treated as described previously with hRenin; we observed a significant stimulation of p-CREB by hRenin and an inhibition of the phosphorylation by YM and YM + PTX, indicating the necessary role of Goq for ATP6AP2 responsiveness (Fig. 10b). c-Jun and VEGF were modulated in a similar way (data not shown). Treatment of NLCs with the intracellular $\mathrm{Ca}^{2+}$ chelator BAPTA-AM (which was added before hRenin) resulted in a significant inhibition of $\mathrm{p}-\mathrm{CREB}$ as well as the release of exosomes, indicating a calcium-dependent mechanism (Fig. 10b).

\section{Suppression of ATP6AP2 by siATP6AP2 in hADSCs inhibits neurogenesis and enhances astrogliogenesis}

Our data indicate that ATP6AP2 plays a key role synergically with CLR-Ms, but its direct role in Neu-Dif remains unclear. Thus we investigated whether ATP6AP2 is essential for neurogenesis of hADSCs: we transfected hADSCs derived from healthy subjects with an ATP6AP2-targeting siRNA (siATP6AP2) in comparison to a negative control siRNA, which has no targeting sequence. Several trials were done to get the high effectiveness of the transfection assay, and the best result was obtained when undifferentiated cells were treated for $48 \mathrm{~h}$ with siRNA (day $-2 / 0$ ) and then were differentiated as previously but with $1 \%$ FBS. We assessed the key factors in these ATP6AP2-knockdown cells (Fig. 11).

Figure 11a shows that mRNA and CLR-M protein levels of ATP6AP2 in cells transfected with siATP6AP2 were, indeed, significantly decreased; siATP6AP2 induced a significant decrease of TUJ-1-positive cells and increase of GFAP-positive cells. Taken together, these data suggest inhibition of neurogenesis and stimulation of astrogliogenesis. Concomitantly, siATP6AP2 abolished completely the FLOT expression in CLR-Ms contrary to CAV which accumulated abundantly. Furthermore, Goq disappeared significantly from CLR-Ms whereas Goi increased.

To further validate the function of ATP6AP2, we checked the expression of the phosphorylated key factors (ERK1/2, CREB, c-Jun and JNK), firstly during the differentiation of siATP6AP2 cells and secondly at day 37 in differentiated siATP6AP2-NLCs to evaluate the renin responsiveness: direct evidence of the impact of ATP6AP2 on ERK/CREB/c-Jun/JNK was observed; in fact, the phosphorylation of ERK1/2 as well as CREB/cJun/JNK decreased significantly during the differentiation of siATP6ATP2-cells and hRenin failed to induce 

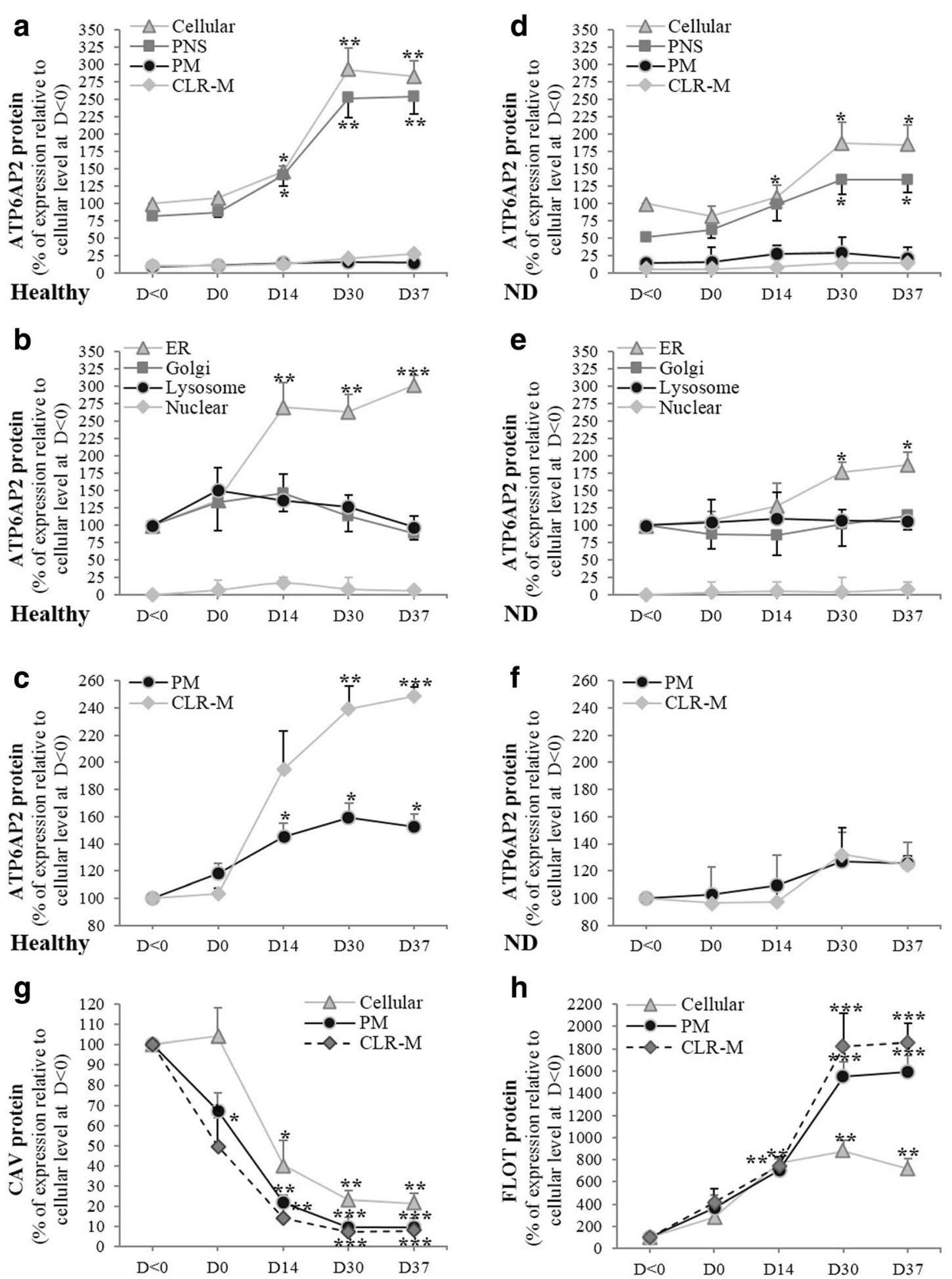

Fig. 6 Subcellular distribution of ATP6AP2 proteins. Subcellular distribution of ATP6AP2 protein in cells derived from healthy (a-c) and ND (d-f) subjects examined during neurogenic differentiation of hADSCs during 37 days of differentiation. Lysate proteins $(50-100 \mu \mathrm{g})$ separated by SDS-PAGE and immunoblotted with polyclonal antibodies directed against full length of ATP6AP2, N-20 recognizes CAV-1 and A-19 epitope mapping within internal region of FLOT-2. Subcellular distribution of ATP6AP2 determined in cellular (total lysates), plasma membrane (PM), caveolae/lipid raft membrane microdomain (CLR-M), postnuclear supernatant (PNS), endoplasmic reticulum (ER), Golgi, lysosome and nucleus. Expression of CAV $(\mathbf{g})$ and FLOT (h) proteins during differentiation of healthy ADSCs. ${ }^{*} P<0.05$, ${ }^{* *} P<0.01 ; * * * P<0.0005$ : D0/14/30/37 vs day < 0 . CAV caveolin, D day, FLOT flotillin, ND neurodegenerative disease 
their phosphorylation in siATP6AP2 NLCs, indicating that the factors are regulated by ATP6AP2. On the other hand, exosome release and the intracellular calcium concentration were inhibited in these cells during differentiation; in fact, when the cells were treated with the PTX and YM (Goi and Goq inhibitors, respectively), PTX did not reverse the inhibitory effects seen in these siATP6AP2 cells; however, YM had significant additional inhibitory effects on exosome release and intracellular calcium, indicating an essential role of Goq in the mechanism of action of ATP6AP2: by silencing the ATP6AP2, Goq is inhibited leading to inhibition of intracellular calcium and exosome release (Fig. 11a).

In Fig. 4, Wnt5a induced markedly p-JNK in response to hRenin in differentiated NLCs derived from healthy donors; here, we treated siATP6AP2 differentiated cells at day 37 with hRenin combined with Wnt5a and evaluated the levels of p-JNK: Wnt5a failed to enhance the hRenin-induced JNK responsiveness in these cells (data not shown). Furthermore, we treated healthy and ND-derived control and siATP6AP2 cells with Wnt5a $24 \mathrm{~h}$ before day 0 and maintained along the Neu-Dif: Wnt5a stimulated p-JNK strongly and significantly in healthy controls but failed to stimulate p-JNK in ND cells. Importantly, in siATP6AP2 cells, p-JNK was completely abolished and Wnt5a did not rescue its phosphorylation, indicating that ATP6AP2 is required for the Wnt5a-induced JNK responsiveness during Neu-Dif (Fig. 11a).

Next, to investigate whether a disruption of CLR-Ms may affect siATP6AP2 knockdown cells and induce any reversibility, and whether CLR-Ms without ATP6AP2 are responsible for the neurogenesis fate, we aimed to deplete membrane sphingomyelin and cholesterol after transfection of the cells with siATP6AP2. So, ADSCs were transfected from day -2 to day 0 with siATP6AP2 and then treated at day 0 with nSMase from $S$. aureus $(1 \mathrm{mU} / \mathrm{ml})$ and $\mathrm{MBCD}(10 \mathrm{nM})$ for $48 \mathrm{~h}$ (Fig. 11b). nSMase treatment of siATP6AP2 knockdown cells depleted CLR-Ms from CAV and induced accumulation of FLOT in these microdomains. However, no additional effects were shown when siATP6AP2 cells were treated with $\mathrm{MBCD}$ in addition to nSMase. Surprisingly, nSMase combined or not with BMCD did not recruit a significant amount of ATP6AP2 into CLR-Ms and did not promote neurogenesis in siATP6AP2-treated cells (no significant variations were observed for TUJ-1positive cells), indicating a necessary role of ATP6AP2 for Neu-Dif and giving evidence that a reversibility in CAV levels in CLR-Ms may occur in cells not expressing ATP6AP2 without impacts on cell differentiation.

Based on these data, there is strong evidence that suppression of ATP6AP2 inhibits neurogenesis of hADSCs and a relocalization of ATP6AP2 into the CLR-Ms could remove the blockage of Neu-Dif. This may indicate that cells having significant high levels of ATP6AP2 in CLRMs with concomitant reduced levels of CAV should have high potential to promote neurogenesis. This has encouraged us to look at whether restoration of ATP6AP2 levels in the CLR-Ms of ADSCs derived from ND patients could re-stimulate their neurogenesis; therefore, we treated ND-hADSCs with nSMase as described previously and observed the following (Fig. 11c): ATP6AP2 accumulated in CLR-Ms where CAV decreased markedly with concomitant induction of the neuronal marker TUJ1; an important reversibility in the

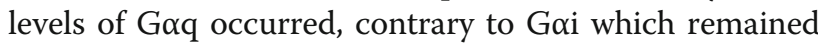
inhibited; a marked increase in the levels of phosphoERK and phospho-JNK was seen; and, in addition, exosome release was induced significantly by nSMase treatment in these cells, where similar positive regulation was obtained in the case of treatment of NDderived cells with nSMase + PTX (the inhibitor of Goi), whereas YM (the inhibitor of Goq) blocked completely the effects of nSMase. These results indicate that depletion of CLR-Ms from CAV, enrichment with ATP6AP2 and Goq activity must be restored in ND-derived cells to induce Neu-Dif.

The dynamic changes in the plasma membrane microdomains led us to consider the importance of membrane fluidity in signal transduction and how it can affect mechanisms, thus we hypothesized that alterations in membrane fluidity may affect ATP6AP2-dependent neurogenesis and contribute to the development of neurological diseases. Cell membrane fluidity of ADSCs was assessed taking into consideration the relocalization of ATP6P2 from membrane to intracellular sites indicating the most important state where ATP6AP2 is no longer retained in the plasma membrane and caveolae. The cells were labeled with DPH probe, and fluorescence anisotropy was determined under conditions where DPH was primarily in the plasma membrane. The results indicate that the fluorescence anisotropy increased significantly when plasma membranes are deprived from ATP6AP2 (GW and siATP6AP2 treatments), indicating that the membrane is more rigid; however, an inverse situation was observed in ATP6AP2-enriched plasma membranes (nSMAse treatment), indicating that the membrane is more fluid which may activate the $\mathrm{G} \alpha$ signaling (Table 3).

\section{Discussion}

ATP6AP2 ((P)RR) has been reported to be involved in neuronal development [40] and in neurogenesis and to be associated with neurological disorders $[6$, 41-43], but no previous study had clarified its role in the neurogenesis fate of hADSCs and its relation with CLR-Ms. In this study, we observed that (P)RR is 


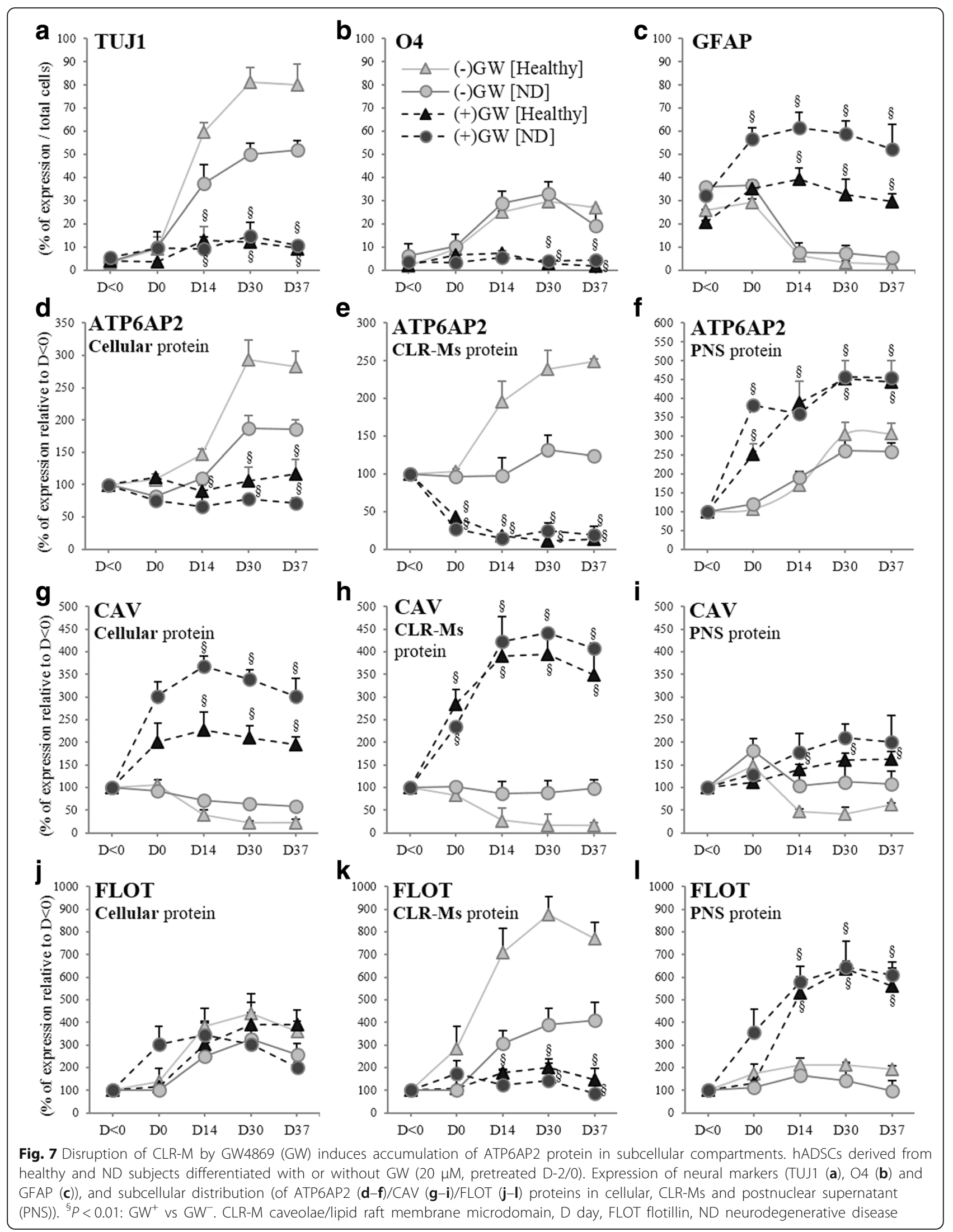


expressed in ADSCs and increased during neural differentiation (contrary to $\mathrm{s}(\mathrm{P}) \mathrm{RR}$ ), localized mainly intracellularly and active in CLR-Ms. Its activity is dependent on the CAV/FLOT balance. In differentiated cells, hRenin-induced (P)RR responsiveness of the MAPK pathway is accentuated by Wnt/PCP

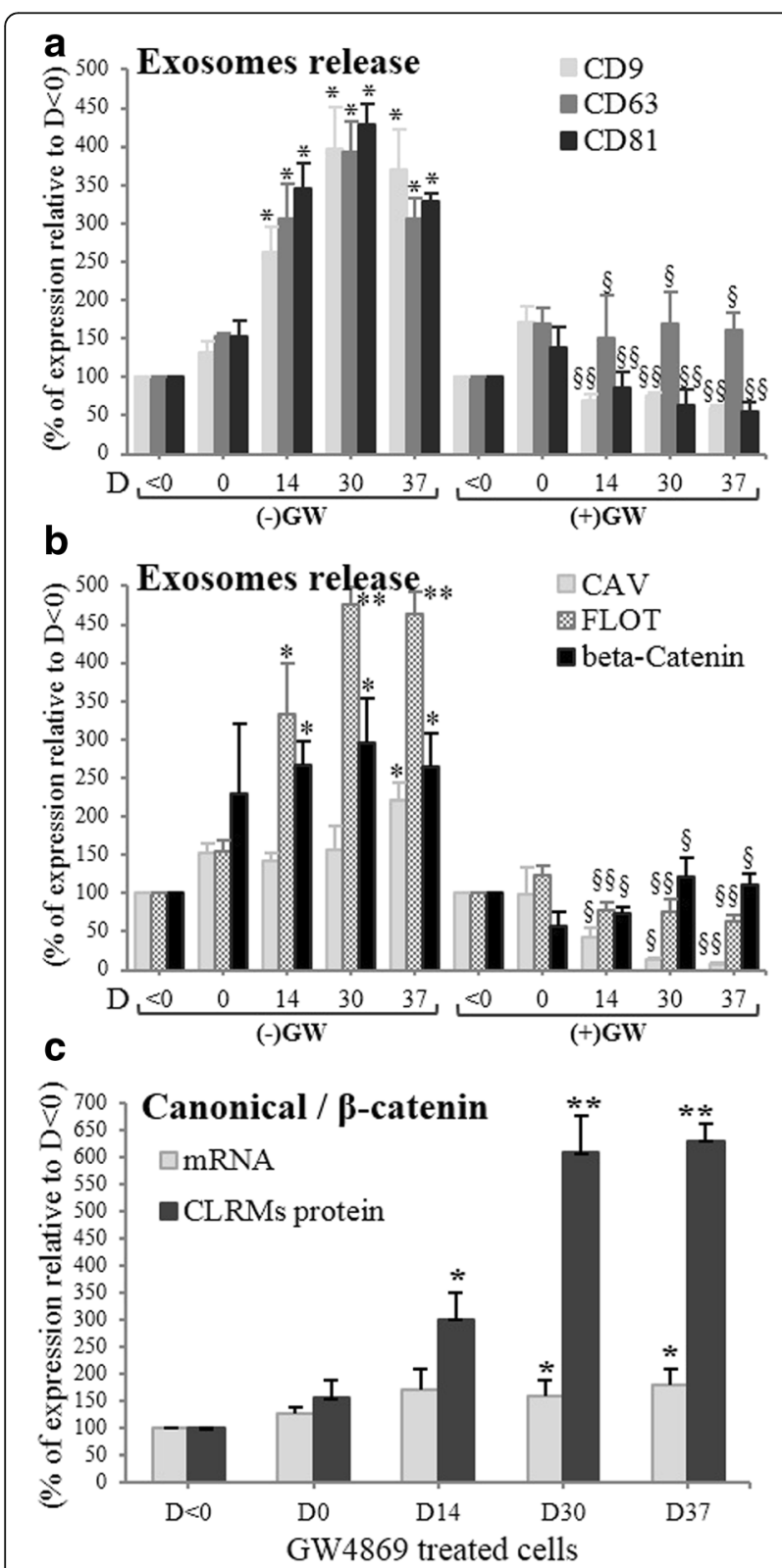

Fig. 8 Disruption of CLR-M by GW4869 (GW) induces reduction of exosome release. hADSCs derived from healthy and ND subjects differentiated with or without GW (20 $\mu \mathrm{M}$, pretreated D-2/0). Exosome fractions analyzed for the assessment of tetraspanins (CD9/63/81) (a) and FLOT/CAV/ $\beta$-catenin (b). Expression of $\beta$-catenin mRNA and protein in CLRMs in GW-treated cells during Neu-Dif (c). ${ }^{*} P$ $<0.01,{ }^{*} P<0.005: D 0 / 14 / 30 / 37$ vs day $<0 ;{ }^{\S} P<0.01,{ }^{\S \S} P<0.005: \mathrm{GW}^{+}$ vs GW'. CAV caveolin, CLRM caveolae/lipid raft membrane microdomain, D day, FLOT flotillin signaling. Concomitantly, a switch from canonical to noncanonical Wnt signaling occurs dependently on exosome release. In addition, $\mathrm{G} \alpha$ proteins crosslink ATP6AP2 to CAV, where a switch from Gai to Goq is necessary for the induction of the differentiation. Knockdown of ATP6AP2 inhibits neurogenesis and a relocalization of ATP6AP2 in CLR-Ms with concomitant reduced levels of CAV promotes neurogenesis.

Interestingly, cells derived from ND patients showed significant reduced levels of ATP6AP2 and downstream signaling. Recently, a meta-analysis of genome-wide association showed an inverse association between ATP6AP2 and multiple sclerosis, Alzheimer's disease and Parkinson's disease [44]. Previous reports have proved expression of ATP6AP2 in the brain and in neuronal cell differentiation $[4,5,34]$, shown decreased levels in XPDS patients [6], shown that conditional depletion induced cognitive impairment and neurodegeneration [7] and reported gene mutation in patients with $\mathrm{X}$ linked mental retardation and epilepsy [42].

Several studies have reported that ATP6AP2 is mainly localized in the ER, with minor concentration on the cell surface $[3,45]$; our results supported these observations and showed an absence in lysosomes/nucleus and a dynamic localization in CLR-Ms. The accumulation of ATP6AP2 in CLR-Ms during Neu-Dif was concomitant with increased levels of FLOTs but decreased levels of CAVs, major CLR-M components. This indicates that cells having high amounts of CAV possess a nondifferentiated phenotype, but cells having high amounts of FLOT and ATP6AP2 possess the potential to differentiate into an NLC phenotype. FLOT is expressed during Neu-Dif [46] and is particularly high in cells that lack CAV (e.g., lymphocytes or neurons) [20]. Interestingly, CAV plays critical roles in regulating neuronal/oligodendral signaling pathways: downregulation of CAV-1 revealed more neuronal and oligodendroglia differentiation but not astrocytes [14, 35, 47], which is in accordance with our data observed with the siATP6AP2 cell model. Three CAV isoforms were expressed in ADMSCs and nearly absent in AD-NLCs, and were shown previously to be expressed in brain endothelial and astroglial cell types [48]. CAVs may exert profound regulatory effects: indeed, CAV can negatively regulate the initiation of intracellular signaling by sequestering membrane signaling proteins (e.g., ATP6AP2, Wnt(s), VATPase, etc.) and preventing their interaction with the appropriate downstream signaling machinery. CAV-1 appears to positively regulate certain classes of signaling ( $\beta$-catenin) [35] and attenuate others (ERK) [13], recruits $\beta$-catenin to caveolae in MDCK cells [49], and inhibits Neu-Dif in NPCs [14] via downregulation of VEGF, pERK, Akt and Stat3 signaling pathways, where VEGF signaling was a crucial target of CAV-1 [14]. Our results 


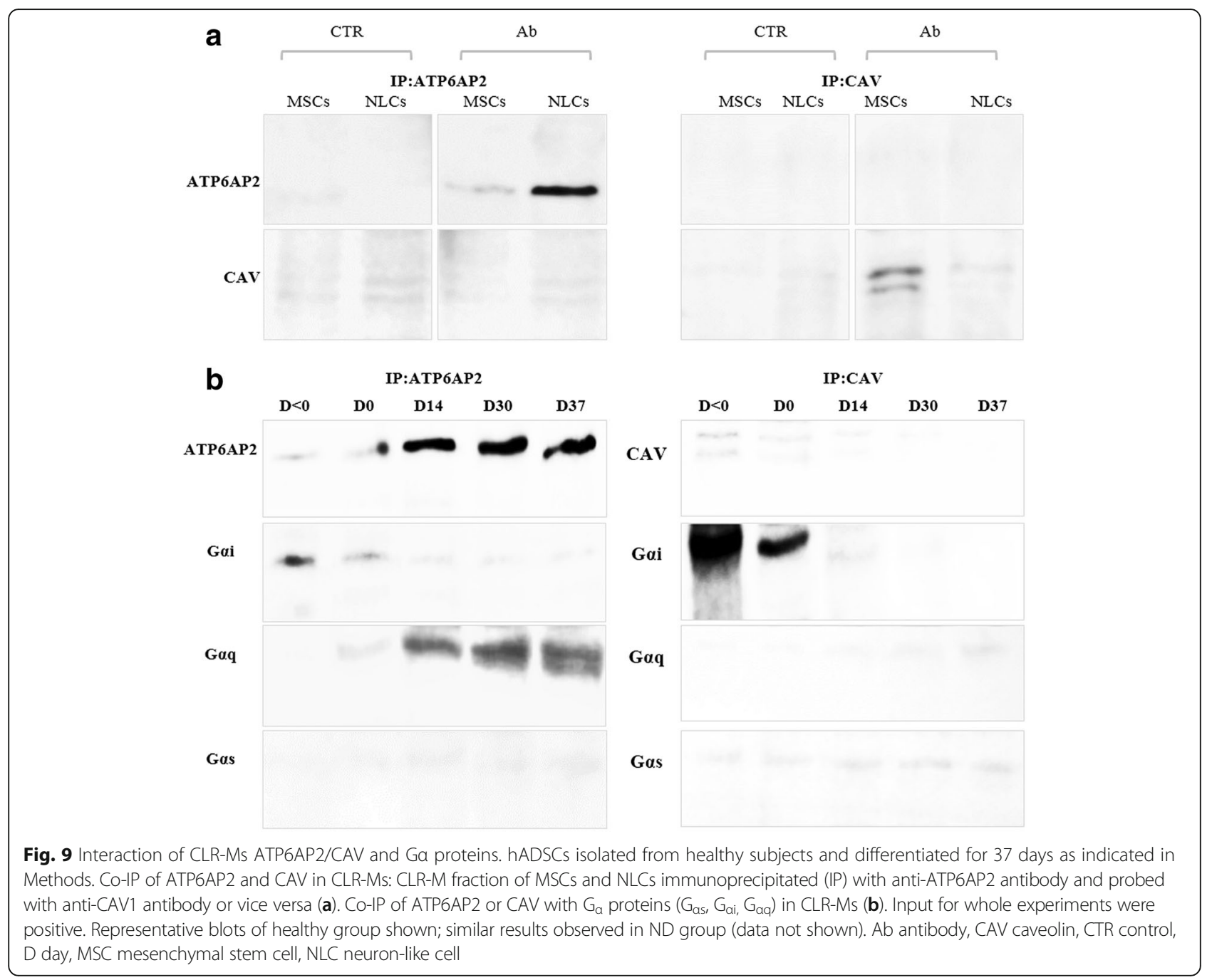

showed increased levels of VEGF mRNA and pERK during neural differentiation, and reduced levels in NDderived cells. In addition, hRenin-induced pERK was completely abolished by the selective inhibitor of MEK1/ 2 kinase PD98059. In fact, previous studies underlie the combination role of ATP6AP2 and the VEGF in some neuropathogeneses [50,51]. The role of VEGF is not limited to that of angiogenesis but also plays a crucial role in neurogenesis $[10,52]$. Importantly, colocalization of ATP6AP2 and VEGF had been reported in hRECs, where hProrenin activates ERK signaling and increases the expression of VEGF mRNA, and the administration of ATP6AP2 blocker (NH2-RIFLKRMPSI-COOH) decreases the expression of VEGF [11]. Moreover, ATP6AP2 interacting with microRNA-152 regulates downstream VEGF expression in hRECs [12]. Interestingly, the mechanism by which ATP6AP2 acts is dependent on $G \alpha$ proteins where an extinction of Gai initiated the differentiation with a crucial role of $G \alpha q$ for ATP6AP2-dependent neurogenesis: a mechanism linking intracellular calcium to exosome release and regulation of CREB and c-Jun, major targets of ERK signaling in neuronal cells. G-proteins relay signals from the plasma membrane to downstream effectors and the physiological functions of $\mathrm{Gq}$ and $\mathrm{Gi}$ have been largely reported previously in neural development and differentiation. Membrane fluidity seems to play an important role and initiate the ATP6AP2 signaling.

The finding that henin failed to induce translocation of $\beta$-catenin into the nucleus in AD-NLCs but induced the phosphorylation of JNK indicates clearly a noncanonical Wnt signaling responsiveness. These results were supported by the observation that Wnt-3a and Dkk1 failed to enhance hRenin response, contrary to Wnt5a. In fact, $\beta$-catenin/LRP-5/LRP-6 (the canonical Wnt components) were highly expressed in MSCs but significantly downregulated in NLCs, contrary to Fzd-3/ PTK-7/VANGL/Celsr(s) (the major noncanonical Wnt/ PCP components). In addition, Wnt-5a and Wnt-7a were markedly increased, contrary to Wnt-3a. 

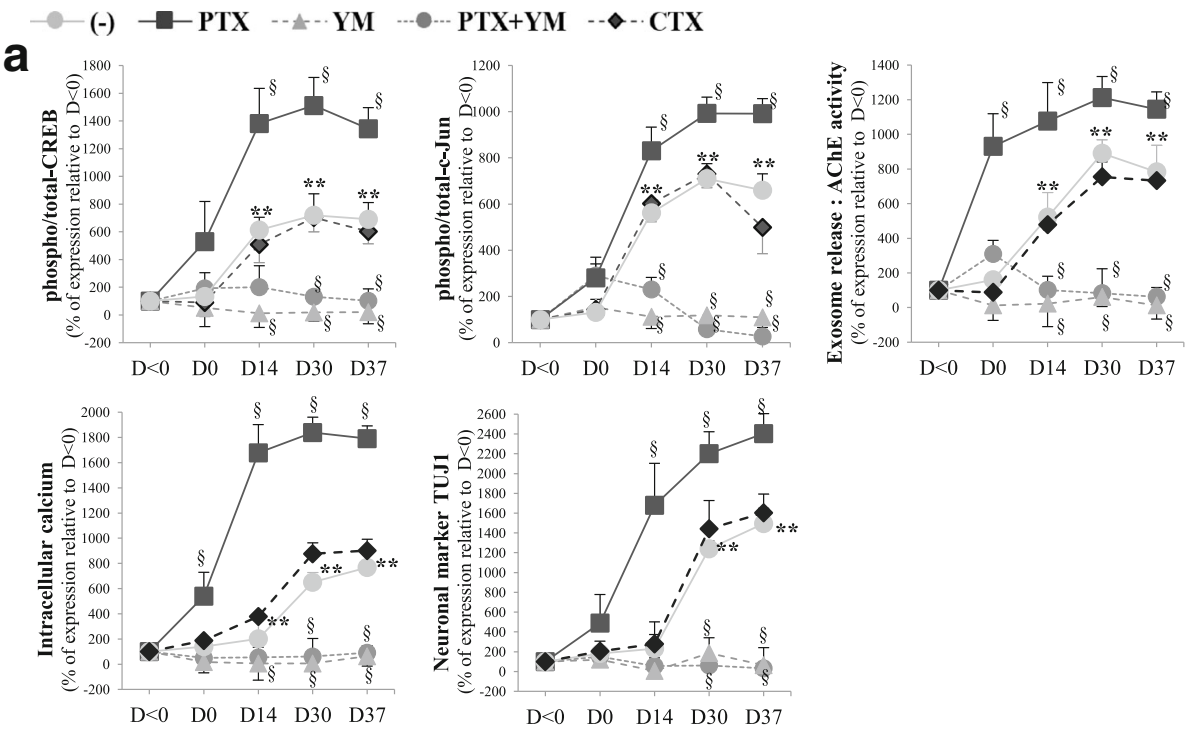

b
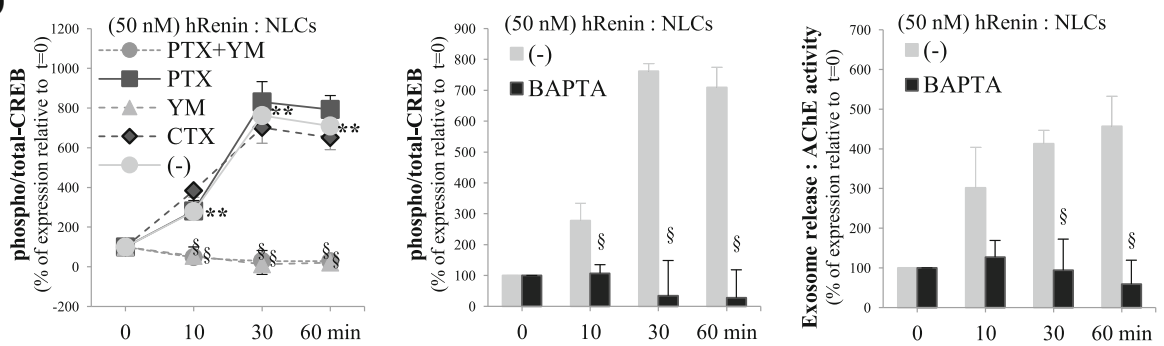

Fig. 10 Activities of $G_{a}$ protein-sensitive CREB/C-Jun, exosome releases and intracellular calcium during neural differentiation of hADSCs. hADSCs isolated from healthy subjects and differentiated for 37 days as indicated in Methods; cells treated for $48 \mathrm{~h}$ (day -2 to day 0 ) with $\mathrm{G}_{\text {as }}$ activator cholera toxin $(C T X, 1 \mu \mathrm{g} / \mathrm{ml})$ or $\mathrm{G}_{\text {aq }}$ protein inhibitor YM254890 (YM, $\left.1 \mu \mathrm{M}\right)$ or $\mathrm{G}_{\text {ai }}$ inhibitor pertussis toxin (PTX, $100 \mathrm{ng} / \mathrm{ml}$ ). Analyses performed along differentiation (a) or at NLC stage (day 37) (b). Nuclear extracts separated by SDS-PAGE and immunoblotted with antibodies directed against total and $\mathrm{p}-\mathrm{CREB}$ and c-Jun. Secreted exosomes collected and quantitated by measuring AChE activity. Involvement of intracellular $\mathrm{Ca}^{2+}$ assessed by treating differentiated NLCs at day 37 with BAPTA-AM $\left(25 \mu \mathrm{M}, 24 \mathrm{~h}\right.$ before adding hRenin) or by measuring $\left[\mathrm{Ca}^{2+}\right]_{\mathrm{i}}$ during Neu-Dif and using Fura2/AM labeling as described in Methods. Neu-Dif evaluated by TUJ1 protein expression. ATP6AP2 responsiveness to hRenin after CTX/PTX/YM treatments evaluated in NLCs at day 37. ${ }^{* *} P<0.01$ : D0/14/30/37 vs day $<0 .{ }^{\S} P<0.01$ : treatments vs control. AChE acetylcholinesterase CREB CAMP response element-binding protein, D day, NLC neuron-like cell

Collectively, these results indicate a significant transition from $\mathrm{Wnt} / \beta$-catenin to $\mathrm{Wnt} / \mathrm{PCP}$ signaling during neural differentiation. Wnt signaling was involved in stem cell differentiation into neural lineages [31], and contradicts recently reported data in adult hippocampal neurogenesis [34] and in embryonic stem cells [53] which showed that $\mathrm{Wnt} / \beta$ catenin signaling blockade promotes neuronal induction and dopaminergic differentiation, and loss of LRP6 increases neuroectodermal differentiation. Furthermore, reduced distribution of LRP6 in the CLR-Ms (where CAV is associated) affects $\beta$-catenin signaling [54] and confirms that CAV binding to LRP6 is required upon Wnt stimulation [55]. ATP6AP2 forms a necessary link between LRP5/6 and Frizzled (Fz) [8].

Our results reveal a role of CLR-Ms in ATP6AP2 signaling, and emphasize the impact of CAV and FLOT.
We previously reported that modulating sphingolipids at cellular levels [27, 56] and in microdomains [27] affects cholesterol, CAV and ERK signaling. Here, depleting sphingolipids from CLR-Ms showed increased expression of ATP6AP2 during neural differentiation, and sphingolipid enrichment reversed this situation. By inhibiting N-SMase with GW, neural differentiation of human ES cells was reversed [57]. In our study, GW inhibits neural differentiation: ATP6AP2 decreased and relocalized concomitantly with FLOT from the CLR-Ms into intracellular compartments, release of exosomes was impaired as observed by a significant reduction in the expression of markers of the tetraspanin family, the reduction of FLOT and CAV recovered in the exosome fraction and there was a reduction in exosome release of $\beta$-catenin. Previous reports showed that purified exosomes are enriched in ceramide and the release of 
a
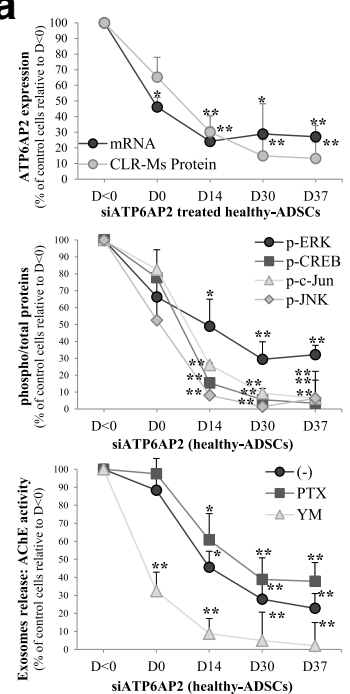

b
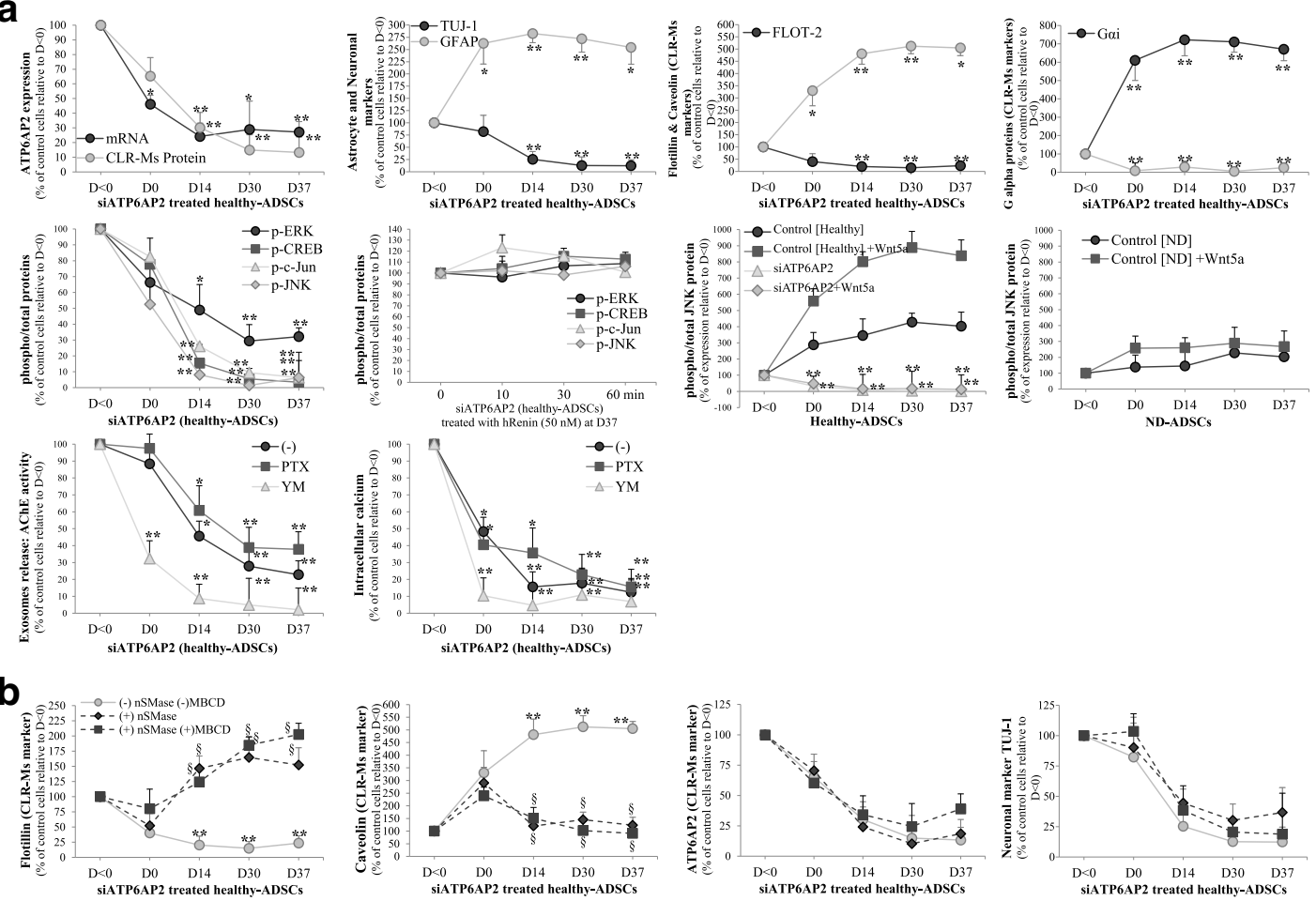

C
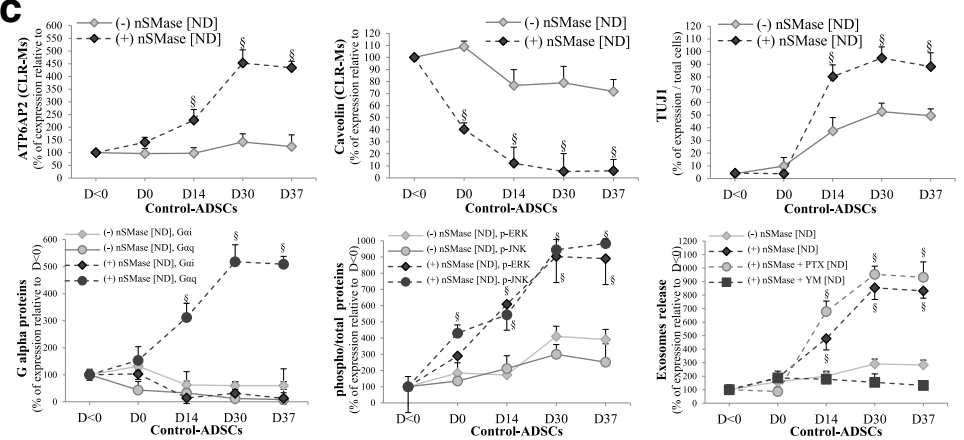

Fig. 11 Inhibition of neurogenesis but enhancement of astrogliogenesis in siATP6AP2 cells and depletion of SM/CL failed to recover ATP6Ap2. $\mathbf{a}, \mathbf{b}$ hADSCs derived from healthy subjects transfected with an ATP6AP2-targeting siRNA (siATP6AP2) for 48 h (day -2/0) and then differentiated with 1\% FBS. a Evaluation of ATP6AP2 mRNA and CLR-M protein levels as well as several key factors during differentiation of ATP6AP2-knockdown cells from day < 0 to day 37: neuronal TUJ1 and astrocyte GFAP markers; CAV and FLOT proteins in CLR-Ms; Gai and Gaq proteins in CLR-Ms; phospho-ERK1/2/ CREB/C-Jun/JNK proteins. Obtained cells at day 37 treated with hRenin (50 nM) for indicated time course to evaluate renin responsiveness. siATP6AP2 cells also treated with $G_{\text {aq }}$ protein inhibitor YM254890 (YM) or $G_{\text {ai }}$ inhibitor pertussis toxin (PTX) as indicated in Fig. 10 to determine their impacts on exosome release and intracellular calcium concentration. $\mathbf{b}$ Impact of CLR-M disruption during siATP6AP2-hADSC differentiation derived from healthy subjects: at day 0, cells were treated with or without nSMase (1 mU/ml) and/or MBCD (10 nM) for 48 h. c Impact of nSMase on hADSCs derived from ND patients during Neu-Dif: ATPAP2 and caveolin proteins in CLR-Ms, expression of neuronal marker TUJ1, Gai and Gaq proteins in CLR-Ms, phospho-ERK/JNK, and exosome release. ${ }^{*} P<0.05,{ }^{* *} P<0.01$ : siATP6AP2 vs control. ${ }^{\S} P<0.01$ : nSMase/MBCD vs control. ADSC adipose-derived mesenchymal stem cell, CAV caveolin, CLR-M caveolae/lipid raft plasma membrane microdomain, CREB CAMP response element-binding protein, D day, ERK extracellular signal-regulated kinase, FLOT flotillin, hRenin human recombinant renin, JNK Jun N-terminal kinase, MBCD methyl- $\beta$-cyclodextrin, ND neurodegenerative disease

exosomes was reduced after the inhibition of N-SMase [58]. A similar profile of results was reported previously where GW reduced exosome release in HEK 293 T cells [22], RAW264.7 macrophages [23] and proinflammatory cytokines [23]. siATP6AP2 knockdown ADSCs promoted astrogenesis but inhibited neuronal and oligodendrocyte differentiation, where ATP6AP2 disappeared from FLOT-deprived/CAV-enriched CLR-Ms: similar to GW-treated cells. These two cell models give evidence of the impact of a mutual association between ATP6AP2 and CLR-Ms on neural differentiation; in fact, disruption of CLR-Ms by GW accumulated sphingolipids/ 
Table 3 Membrane fluidity assessment

\begin{tabular}{llll}
\hline & $\begin{array}{l}\text { Fluorescence } \\
\text { anisotropy }\end{array}$ & $\begin{array}{l}\text { Percentage } \\
\text { of control }\end{array}$ & $P$ value \\
\hline MSCs & & & \\
Control (untreated cells) & $0.184 \pm 0.012$ & 100 & \\
GW & $0.215 \pm 0.019$ & 116.8 & $<0.05$ \\
nSMase & $0.148 \pm 0.008$ & 80.4 & $<0.01$ \\
siAT6AP2 & $0.219 \pm 0.010$ & 119.0 & $<0.01$ \\
NLCS & & & \\
Control (untreated cells) & $0.167 \pm 0.009$ & 100 & \\
GW & $0.208 \pm 0.011$ & 124.5 & $<0.01$ \\
nSMase & $0.150 \pm 0.018$ & 89.8 & $\mathrm{NS}$ \\
siAT6AP2 & $0.203 \pm 0.007$ & 121.6 & $<0.01$ \\
\hline
\end{tabular}

The cells derived from adipose tissue of healthy subjects were labeled with 1,6-diphenyl-1,3,5-hexatriene, and the fluorescence anisotropy of the probe was determined. The measurements were performed at $37^{\circ} \mathrm{C}$ for $2 \mathrm{~min}$ immediately after addition of the fluorescent probe. Results presented as mean \pm SEM

$P<0.05$ and $P<0.001$, treated cells compared with control cells GW GW4869, MSC mesenchymal stem cell, NLC neural-like cell, NS not significant

CAV in CLR-Ms but deprived them of ATP6AP2; and ATP6AP2 knockdown cells deprived CLR-Ms of ATP6AP2 but accumulated CAV. However, only the data obtained from nSMase-treated siATP6AP2 cells give strong evidence of the main role of ATP6AP2 in Neu-Dif to be maintained in CLR-Ms for neuronal induction. In fact, when siATP6AP2 cells are treated with nSMase, recovery of FLOT in CLR-Ms and depletion of CAV from CLR-Ms were observed without induction of the Neu-Dif, indicating that it is essential to relocate ATP6AP2 in CLRMs for neurogenesis.
In conclusion, this study shows that ATP6AP2 plays an important role in neural differentiation of ADSCs, and its activity involves the Wnt signaling pathways and $\mathrm{G} \alpha$ proteins in a way dependent on the dynamics of the membrane microdomains affecting the release of exosomes, thus giving evidence that its intracellular accumulation may be determinant for the induction of neuronal and oligodendrocyte differentiation. Our results provide an acceptable promise to use hADSCs as a proper autologous adult stem cell population for cell replacement therapy of neurological disorders, but highlight the importance in the selection of cells that must have higher amounts of ATP6AP2 particularly in CLRMs for better response, thus emphasizing the crucial role of CLR-Ms. For this purpose, further studies are required to test in vivo the hypothesis that in neurodegenerative disorders the autologous transplantation of undifferentiated stem cells must be considered carefully or instead will be needed prior to induction of neural differentiation. The role of ATP6AP2-over/underexpressed ADSCs should also be clarified during differentiation into other lineages of cells (hepatocytes, nephron progenitor cells, cardiomyocytes, etc.) in a perspective of cell therapy; for example, cardiovascular diseases where hypertension and hypercholesterolemia were correlated with the RAS components $[59,25]$, which in turn were associated with ATP6AP2.

\section{Conclusion}

The novel observation of this report is that ATP6AP2 is associated with CLR-Ms and regulates the composition of the membrane microdomains, affecting membrane fluidity and mediating different Wnt signaling pathways

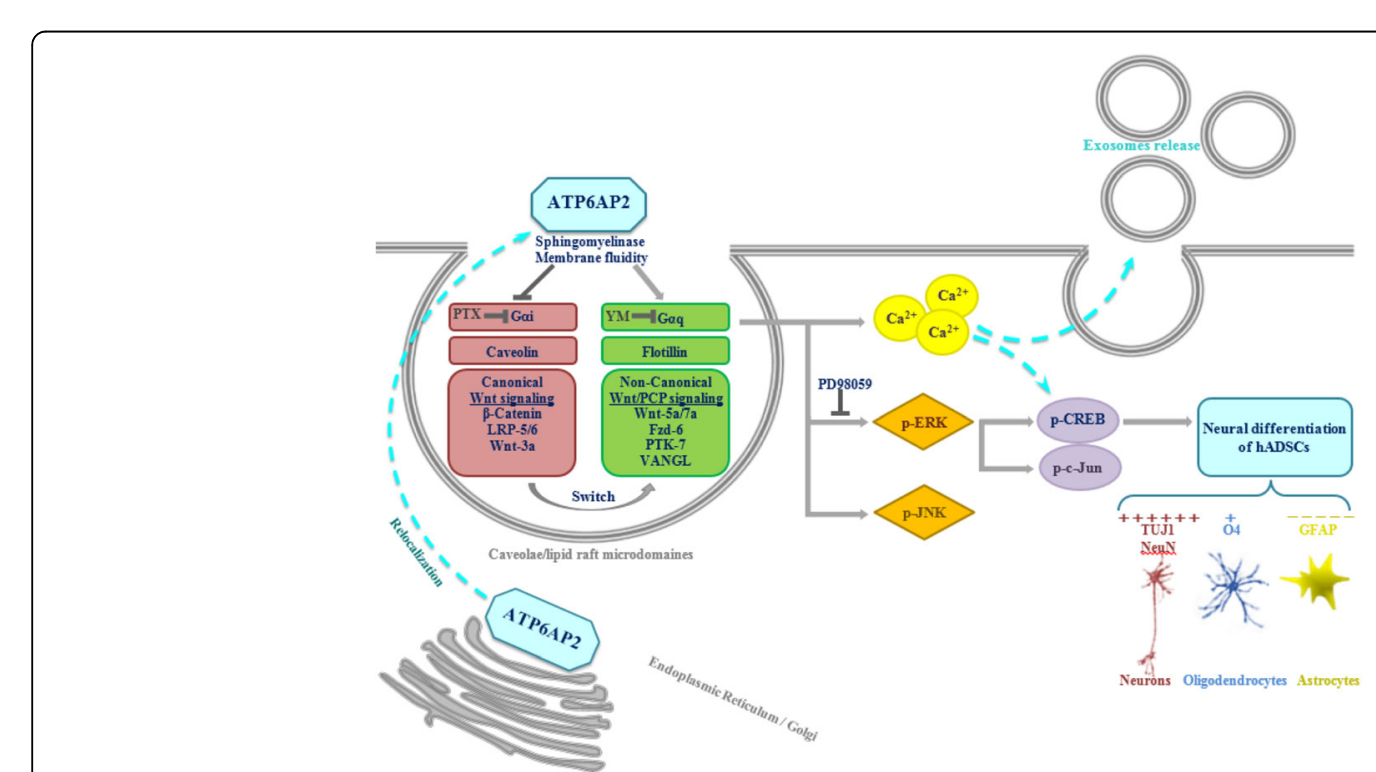

Fig. 12 Model for regulation of ATP6AP2-induced neural differentiation by Wnt5a/Gaq/ERK/JNK/C-Jun/CREB and calcium-dependent exosome release signaling pathway 
by a mechanism which is dependent on a switch from

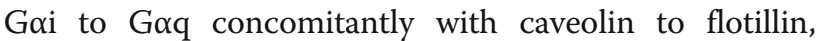
canonical to PCP (attenuation of $\beta$-catenin/LRP-5/LRP6/Wnt-3a, increase of Fzd-6/PTK-7/VANGL/Celsr1-3/ Wnt-5a/Wnt-7a, unchanged Fzd-3/Wnt-4a/Wnt-11) and intracellular $\mathrm{Ca}^{2+}$-dependent release of exosomes (Fig. 12). hRenin stimulates ATP6AP2 to induce p-ERK/JNK/VEGF/CREB/C-Jun and exosome release. ADSCs are competent to differentiate into neurons when CLR-Ms are enriched in ATP6AP2 and deprived of caveolin. Knockdown of ATP6AP2 which deprives CLR-Ms of ATP6AP2 inhibits neurogenesis but induces astrogenesis, and caveolin depletion did not recover the inhibition of Neu-Dif. Physiological relevance is found in patients with neurological disorders.

\section{Additional files}

Additional file 1: Table SI1. Markers of nuclear and microsomal proteins in nuclear, microsomal and CLR-M fractions. (DOCX 75 kb)

Additional file 2: Table SI2. Primer sequences used for quantitative RT-PCR. (DOCX $78 \mathrm{~kb}$ )

\section{Abbreviations}

(P)RR : Prorenin receptor; AChE: Acetylcholinesterase; AD: Alzheimer's disease; ADSC: Adipose-derived mesenchymal stem cell; ALS: Amyotrophic lateral sclerosis; CAV: Caveolin; CD: Cluster of differentiation; CLR-M: Caveolae/lipid raft plasma membrane microdomain; CREB: CAMP response element-binding protein; DPH: 1,6-Diphenyl-1,3,5-hexatriene; ER: Endoplasmic reticulum: ERK: Extracellular signal-regulated kinase; FLOT: Flotillin; GAPDH: Glyceraldehyde 3-phosphate dehydrogenase; IP: Immunoprecipitate; JNK: Jun N-terminal kinase; MAPK: Mitogen-activated protein kinase; MLR: Microdomain lipid raft; MS: Multiple sclerosis; MSC: Mesenchymal stem cell; NB: Neurobasal; N-CLR-M: Noncaveolae/nonlipid raft plasma membrane microdomains; ND: Neurodegenerative disease; Neu-Dif: Neuronal differentiation; NLC: Neuron-like cell; NSC: Neural stem cell; NSP: Neurosphere-like structures; PBMC: Peripheral blood mononuclear cell; PD: Parkinson disease; PEDF: Pigment epithelium-derived factor; PM: Plasma membrane; PSA: Penicilin-stryptomycin-amphotericim; RAS: Reninangiotensin system; SVF: Stromal vascular fraction; TGN: Trans-Golgi network; VEGF: Vascular endothelial growth factor; Wnt: Wingless-type MMTV integration site family member

\section{Acknowledgements}

The authors would like to acknowledge all parties that participated in this study. This work has been funded with support from the Lebanese University and the CEDRE from the French MENESR. The authors thank Dr. Rihab Nasr (AUBMC) for her assistance in fluorescence microscopy analysis.

\section{Funding}

This work was supported by grants from the Lebanese University and the CEDRE from the French MENESR (35399WH).

\section{Availability of data and materials}

All data generated or analyzed during this study are included in this published article and its additional files.

\section{Authors' contributions}

NM contributed to conception, study design and conducting the study. NM, $\mathrm{KH}, J \mathrm{DA}, \mathrm{DC}, \mathrm{BI}$ and $\mathrm{GO}$ contributed to experimentation and data collection. $\mathrm{NM}, \mathrm{ZF}, \mathrm{KH}$ and JB contributed to data analysis and interpretation. NM, JB and ZF contributed to manuscript writing and editing. IA, DA and DQ contributed to patient selection and sample procurement. AA contributed to management. All authors read and approved the final manuscript.

\section{Ethics approval and consent to participate}

All clinical investigations have been conducted according to the principles expressed in the Declaration of Helsinki (https://www.wma.net/policies-post/ wma-declaration-of-helsinki-ethical-principles-for-medical-research-involvinghuman-subjects/) as revised in 2008 (https:/www.wma.net/wp-content/ uploads/2016/11/DoH-Oct2008.pdf). All subjects gave written informed consent. Fresh samples were procured by Dr. Ibrahim El Achkar and frozen samples were procured by the Nancy University Hospital Clinical Investigation Center (ethical approval number, CPP3.07.01, France).

\section{Competing interests}

The authors declare that they have no competing interests.

\section{Publisher's Note}

Springer Nature remains neutral with regard to jurisdictional claims in published maps and institutional affiliations.

\section{Author details}

${ }^{1}$ Department of Biology, Lebanese University, Faculty of Sciences III, Kobbe, Lebanon. ${ }^{2}$ Doctoral School for Sciences and Technology, Azm Center for the Research in Biotechnology and its Applications, Lebanese University, Tripoli, Lebanon. ${ }^{3}$ Doctoral School for Sciences and Technology, Faculty of Sciences I, Lebanese University, Hadath, Lebanon. ${ }^{4}$ Reviva Regenerative Medicine Center, Human Genetic Center, Middle East Institute of Health Hospital, Bsalim, Lebanon. ${ }^{5}$ Ayoub Clinic Lebanon and Department of Neuroloradiology, Limoges University Hospital, EA3842 Limoges, Lebanon. ${ }^{6}$ Achkar Clinics, St. Elie Center, Antelias, Lebanon.

${ }^{7}$ Diabetologia-Endocrinology \& Nutrition, CHRU Nancy, INSERM 954,

University Henri Poincaré, Faculty of Medicine, Nancy, France.

Received: 15 January 2018 Revised: 19 March 2018

Accepted: 4 April 2018 Published online: 11 May 2018

\section{References}

1. Matsushita K, Wu Y, Okamoto Y, et al. Local renin angiotensin expression regulates human mesenchymal stem cell differentiation to adipocytes. Hypertension. 2006;48(6):1095-102.

2. Jansen $E$, Martens $G$. Novel insights into V-ATPase functioning: distinct roles for its accessory subunits ATP6AP1/Ac45 and ATP6AP2/(pro) renin receptor. Curr Protein Pept Sci. 2012:13(2):124-33.

3. Nguyen $\mathrm{G}$, Delarue F, Burckle C, et al. Pivotal role of the renin/prorenin receptor in angiotensin II production and cellular responses to renin. J Clin Invest. 2002;109(11):1417-27.

4. Shan Z, Cuadra A, Sumners $C$, et al. Characterization of a functional (pro)renin receptor in rat brain neurons. Exp Physiol. 2008;93(5):701-8.

5. Contrepas A, Walker J, Koulakoff A, et al. A role of the (pro)renin receptor in neuronal cell differentiation. Am J Physiol Regul Integr Comp Physiol. 2009; 297(2):R250-7.

6. Korvatska O, Strand N, Berndt J, et al. Altered splicing of ATP6AP2 causes X-linked parkinsonism with spasticity (XPDS). Hum Mol Genet. 2013;22(16): 3259-68.

7. Dubos A, Castells-Nobau A, Meziane $H$, et al. Conditional depletion of intellectual disability and Parkinsonism candidate gene ATP6AP2 in fly and mouse induces cognitive impairment and neurodegeneration. Hum Mol Genet. 2015:24(23):6736-55.

8. Cruciat C, Ohkawara B, Acebron S, et al. Requirement of prorenin receptor and vacuolar H+-ATPase-mediated acidification for Wnt signaling. Science. 2010;327(5964):459-63.

9. Beckermann B, Kallifatidis G, Groth A, et al. VEGF expression by mesenchymal stem cells contributes to angiogenesis in pancreatic carcinoma. Br J Cancer. 2008;99(4):622-31.

10. Han J, Calvo C, Kang T, et al. Vascular endothelial growth factor receptor 3 controls neural stem cell activation in mice and humans. Cell Rep. 2015; 10(7):1158-72.

11. Kanda A, Noda K, Saito W, Ishida S. (Pro)renin receptor is associated with angiogenic activity in proliferative diabetic retinopathy. Diabetologia. 2012; 55(11):3104-13. 
12. Haque $R$, Hur E, Farell A, et al. MicroRNA-152 represses VEGF and TGF $\beta 1$ expressions through post-transcriptional inhibition of (Pro)renin receptor in human retinal endothelial cells. Mol Vis. 2015;21:224-35.

13. Stern C, Mermelstein P. Caveolin regulation of neuronal intracellular signaling. Cell Mol Life Sci. 2010;67(22):3785-95.

14. Li Y, Luo J, Lau W, et al. Caveolin-1 plays a crucial role in inhibiting neuronal differentiation of neural stem/progenitor cells via VEGF signaling-dependent pathway. PLoS One. 2011;6(8):e22901.

15. Galbiati F, Volonte D, Gil O, et al. Expression of caveolin-1 and -2 in differentiating PC12 cells and dorsal root ganglion neurons: caveolin-2 is up-regulated in response to cell injury. Proc Natl Acad Sci U S A. 1998;95(17):10257-62.

16. Zschocke J, Manthey D, Bayatti N, et al. Estrogen receptor alpha-mediated silencing of caveolin gene expression in neuronal cells. J Biol Chem. 2002; 277(41):38772-80

17. Boulware M, Kordasiewicz H, Mermelstein P. Caveolin proteins are essential for distinct effects of membrane estrogen receptors in neurons. J Neurosci. 2007;27(37):9941-50

18. D'Orlando C, Guzzi F, Gravati M, et al. Retinoic acid- and phorbol esterinduced neuronal differentiation down-regulates caveolin expression in GnRH neurons. J Neurochem. 2008;104(6):1577-87.

19. Egawa J, Pearn M, Lemkuil B, et al. Membrane lipid rafts and neurobiology: age-related changes in membrane lipids and loss of neuronal function. J Physiol. 2016;594(16):4565-79.

20. Lang D, Lommel S, Jung M, et al. Identification of Reggie-1 and Reggie-2 as plasma membrane-associated proteins which cocluster with activated GPIanchored cell adhesion molecules in non-caveolar micropatches in neurons. J Neurobiol. 1998;37(4):502-23.

21. Stuermer C, Lang D, Kirsch D, et al. Glycosylphosphatidyl inositol-anchored proteins and fyn kinase assemble in noncaveolar plasma membrane microdomains defined by Reggie-1-2. Mol Biol Cell. 2001;12(10):3031-45.

22. Chairoungdua A, Smith D, Poschard P, et al. Exosome release of $\beta$-catenin: a novel mechanism that antagonizes Wnt signaling. J Cell Biol. 2010;190(6):1079-91.

23. Essandoh $K$, Yang L, Wang $X$, et al. Blockade of exosome generation with GW4869 dampens the sepsis-induced inflammation and cardiac dysfunction. Biochim Biophys Acta. 2015;1852(11):2362-71.

24. Yoshinaka K, Kumanogoh H, Nakamura S, et al. Identification of V-ATPase as a major component in the raft fraction prepared from the synaptic plasma membrane and the synaptic vesicle of rat brain. Neurosci Lett. 2004;363(2):168-72.

25. AlBacha J, Khoury M, Mouawad C, et al. High incidence of ACE/PAl-1 in association to a spectrum of other polymorphic cardiovascular genes involving PBMCs proinflammatory cytokines in hypertensive hypercholesterolemic patients: reversibility with a combination of ACE inhibitor and statin. PLoS One. 2015;10(5):e0127266.

26. Alio Del Barrio J, El Zarif M, de Miguel M, et al. Cellular therapy with human autologous adipose-derived adult stem cells for advanced keratoconus. Cornea. 2017;36(8):952-60.

27. Makdissy N, Haddad K, Mouawad C, et al. Regulation of SREBPs by sphingomyelin in adipocytes via a caveolin and Ras-ERK-MAPK-CREB Signaling pathway. PLoS One. 2015;10(7):e0133181.

28. Théry C, Amigorena S, Raposo G, et al. Isolation and characterization of exosomes from cell culture supernatants and biological fluids. Curr Protoc Cell Biol. 2006;3:3.22. https://doi.org/10.1002/0471143030.cb0322s30.

29. Lu X, Wang F, Xu C, et al. Soluble (pro)renin receptor via beta-catenin enhances urine concentration capability as a target of liver $X$ receptor. Proc Natl Acad Sci U S A. 2016;113:E1898-906.

30. Feldt S, Maschke U, Dechend $\mathrm{R}$, et al. The putative (pro)renin receptor blocker HRP fails to prevent (pro)renin signaling. J Am Soc Nephrol. 2008;19(4):743-8.

31. Lange C, Mix E, Rateitschak K, et al. Wnt signal pathways and neural stem cell differentiation. Neurodegener Dis. 2006;3(1-2):76-86.

32. Yamanaka $\mathrm{H}$, Moriguchi $\mathrm{T}$, Masuyama $\mathrm{N}$, et al. JNK functions in the noncanonical Wnt pathway to regulate convergent extension movements in vertebrates. EMBO Rep. 2002;3(1):69-75.

33. Wang C, Zhao Y, Su Y, et al. C-Jun N-terminal kinase (JNK) mediates Wnt5ainduced cell motility dependent or independent of RhoA pathway in human dental papilla cells. PLoS One. 2013;8(7):e69440

34. Schafer $S$, Han J, Pena M, et al. The Wnt adaptor protein ATP6AP2 regulates multiple stages of adult hippocampal neurogenesis. J Neurosci. 2015;35(12): 4983-98.

35. Li Y, Lau W, So K, et al. Caveolin-1 inhibits oligodendroglial differentiation of neural stem/progenitor cells through modulating $\beta$-catenin expression. Neurochem Int. 2011;59(2):114-21.
36. Lachenal G, Pernet-Gallay K, Chivet M, et al. Release of exosomes from differentiated neurons and its regulation by synaptic glutamatergic activity. Mol Cell Neurosci. 2011;46(2):409-18.

37. Fauré J, Lachenal G, Court M, et al. Exosomes are released by cultured cortical neurones. Mol Cell Neurosci. 2006;31(4):642-8.

38. Krämer-Albers $\mathrm{E}$, Bertz $\mathrm{N}$, Tenzer $\mathrm{S}$, et al. Oligodendrocytes secrete exosomes containing major myelin and stress-protective proteins: trophic support for axons? Proteomics Clin Appl. 2007;1(11):1446-61.

39. Taylor A, Robinson M, Gifondorwa D, et al. Regulation of heat shock protein 70 release in astrocytes: role of signaling kinases. Dev Neurobiol. 2007;67(13):1815-29.

40. Nguyen G. The (pro)renin receptor in health and disease. Ann Med. 2010; 42(1):13-8.

41. Poorkaj P, Raskind W, Leverenz J, et al. A novel X-linked four-repeat tauopathy with Parkinsonism and spasticity. Mov Disord Off J Mov Disord Soc. 2010;25(10):1409-17.

42. Ramser J, Abidi F, Burckle C, et al. A unique exonic splice enhancer mutation in a family with $\mathrm{X}$-linked mental retardation and epilepsy points to a novel role of the renin receptor. Hum Mol Genet. 2005;14(8):1019-27.

43. Hedera P, Alvarado D, Beydoun A, et al. Novel mental retardation-epilepsy syndrome linked to Xp21.1-p11.4. Ann Neurol. 2002;51(1):45-50.

44. Goldstein B, Speth R, Trivedi M. Renin-angiotensin system gene expression and neurodegenerative diseases. JRAAS. 2016;17(3) https://doi.org/10.1177/1470320316666750.

45. Sihn G, Burckle C, Rousselle A, et al. (Pro)renin receptor: subcellular localizations and functions. Front Biosci (Elite Ed). 2013;5:500-8. https://doi.org/10.2741/E631.

46. Volonté D, Galbiati F, Li S, et al. Flotillins/cavatellins are differentially expressed in cells and tissues and form a hetero-oligomericcomplex with caveolins in vivo. Characterization and epitope-mapping of a novel flotillin-1 monoclonalantibody probe. J Biol Chem. 1999;274(18):12702-9.

47. Wang S, Kan Q, Sun Y, et al. Caveolin-1 regulates neural differentiation of rat bone mesenchymal stem cells into neurons by modulating Notch signaling. Int J Dev Neurosci. 2013:31(1):30-5.

48. Ikezu T, Ueda H, Trapp B, et al. Affinity-purification and characterization of caveolins from the brain: differential expression of caveolin-1, -2, and -3 in brain endothelial and astroglial cell types. Brain Res. 1998;804(2):177-92.

49. Galbiati F, Volonte D, Brown A, et al. Caveolin-1 expression inhibits Wnt/ beta-catenin/Lef-1 signaling by recruiting beta-catenin to caveolae membrane domains. J Biol Chem. 2000;275(30):23368-77.

50. Fournier N, Duman R. Role of vascular endothelial growth factor in adult hippocampal neurogenesis: implications for the pathophysiology and treatment of depression. Behav Brain Res. 2012;227(2):440-9.

51. Sun $Y$, Jin $K$, Xie $L$, et al. VEGF-induced neuroprotection, neurogenesis, and angiogenesis after focal cerebral ischemia. J Clin Invest. 2003:111(12):1843-51.

52. Jin K, Zhu Y, Sun Y, et al. Vascular endothelial growth factor (VEGF) stimulates neurogenesis in vitro and in vivo. Proc Natl Acad Sci U S A. 2002:99(18):11946-50.

53. Čajánek L, Ribeiro D, Liste I, et al. Wnt/ $\beta$-catenin signaling blockade promotes neuronal induction and dopaminergic differentiation in embryonic stem cells. Stem Cells. 2009;27(12):2917-27.

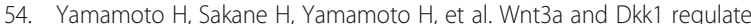
distinct internalization pathways of LRP6 to tune the activation of betacatenin signaling. Dev Cell. 2008;15(1):37-48.

55. Yamamoto H, Komekado H, Kikuchi A. Caveolin is necessary for Wnt-3adependent internalization of LRP6 and accumulation of beta-catenin. Dev Cell. 2006:11(2):213-23.

56. Al-Makdissy N, Younsi M, Pierre S, et al. Sphingomyelin/cholesterol ratio: an important determinant of glucose transport mediated by GLUT-1 in 3T3-L1 preadipocytes. Cell Signal. 2003;15(11):1019-30.

57. He Q, Wang G, Wakade $\mathrm{S}$, et al. Primary cilia in stem cells and neural progenitors are regulated by neutral sphingomyelinase 2 and ceramide. Mo Biol Cell. 2014:25(11):1715-29.

58. Trajkovic K, Hsu C, Chiantia S, et al. Ceramide triggers budding of exosome vesicles into multivesicular endosomes. Science. 2008;319(5867):1244-7.

59. Lu H, Rateri D, Feldman D, et al. Renin inhibition reduces hypercholesterolemiainduced atherosclerosis in mice. J Clin Invest. 2008;118(3):984-93. 